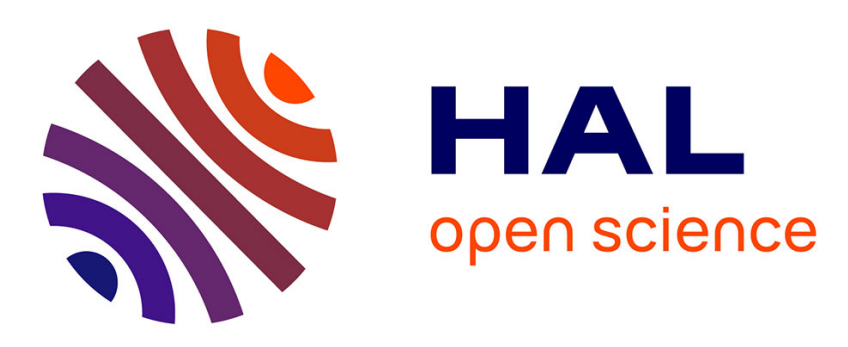

\title{
An essential model for generating walking motions for humanoid robots
}

Víctor De-León-Gómez, Qiuyue Luo, Anne Kalouguine, J. Alfonso Pámanes, Yannick Aoustin, Christine Chevallereau

\section{- To cite this version:}

Víctor De-León-Gómez, Qiuyue Luo, Anne Kalouguine, J. Alfonso Pámanes, Yannick Aoustin, et al.. An essential model for generating walking motions for humanoid robots. Robotics and Autonomous Systems, 2019, 112, pp.229-243. 10.1016/j.robot.2018.11.015 . hal-01990079

\section{HAL Id: hal-01990079 \\ https://hal.science/hal-01990079}

Submitted on 22 Jan 2019

HAL is a multi-disciplinary open access archive for the deposit and dissemination of scientific research documents, whether they are published or not. The documents may come from teaching and research institutions in France or abroad, or from public or private research centers.
L'archive ouverte pluridisciplinaire HAL, est destinée au dépôt et à la diffusion de documents scientifiques de niveau recherche, publiés ou non, émanant des établissements d'enseignement et de recherche français ou étrangers, des laboratoires publics ou privés. 


\title{
An essential model for generating walking motions for humanoid robots
}

\author{
Víctor De-León-Gómez ${ }^{\mathrm{a}, *}$, Qiuyue Luo ${ }^{\mathrm{a}}$, Anne Kalouguine $^{a, \mathrm{~b}}$, J. Alfonso \\ Pámanes $^{\mathrm{c}}$, Yannick Aoustin ${ }^{\mathrm{a}}$, Christine Chevallereau ${ }^{\mathrm{a}}$ \\ ${ }^{a}$ Laboratoire des Sciences du Numérique de Nantes (LS2N) UMR CNRS 6004 \\ 1 rue de la Noë 44300, Nantes, France. \\ ${ }^{b}$ Sotfbank Robotics, 43 rue du Colonel Pierre Avia, 75015 Paris, France \\ ${ }^{c}$ TecNM/Instituto Tecnológico de la Laguna. Blvd. Revolución y \\ Cuauhtémoc S/N, C.P. 27000, Torreón, Coah., México
}

\begin{abstract}
The modeling of humanoid robots with many degrees-of-freedom (DoF) can be done via the complete dynamic model. However, the complexity of the model can hide the essential factor of the walking, i.e. the equilibrium of the robot. One alternative is to simplify the model by neglecting some dynamical effects like in the 3D Linear Inverted Pendulum (LIP) model. Nonetheless, the assumption that the ZMP will be at the base of the pendulum is not ensured and the resulting walking gaits can make the Zero Moment Point (ZMP) evolves outside of the convex hull of support when they are replicated by the complete model of any humanoid robot. The objective of this paper is to propose a new model for walking that has the same dimensions as the 3D LIP model but considers the complete dynamics of the humanoid. The proposed model is called essential model and it can be written based on the internal states of the robot and/or external information, thereby generating models for different purposes. The main advantage of the essential model is that it allows to generate walking gaits that ensure that the Zero Moment Point (ZMP) is kept in a desired position or
\end{abstract}

\footnotetext{
${ }^{*}$ Corresponding author

Email addresses: Victor.Deleongomez@ls2n.fr, vsdeleon@hotmail.com (Víctor De-León-Gómez), Qiuyue.Luo@ls2n.fr (Qiuyue Luo), anne.kalouguine@softbankrobotics.com (Anne Kalouguine ${ }^{a,}$ ), alfonso.pamanes@gmail.com (J. Alfonso Pámanes), yannick.aoustin@ls2n.fr (Yannick Aoustin), christine.chevallereau@ls2n.fr (Christine Chevallereau)
}

Preprint submitted to Robotics and Autonomous Systems

December 20, 2018 
it follows a desired path while the gait is performed. Furthermore, impacts of the swing foot with the ground can be considered to compute periodic walking gaits. In order to show the advantages of the proposed model, numerical studies are performed to design periodic walking gaits for the humanoid robot ROMEO. Keywords: Modelling, Biped walking, Periodic motions, Humanoid robots

\section{Introduction}

In the design of walking gaits for humanoid robots a simple model is often desired. However, the simpler the model, the more inaccurate the solution that describes the behavior of the physical system. In spite of this weakness, 5 the 3D Linear inverted pendulum (LIP) model is still the most used model to develop 3D walking gaits due to its simplicity and the fact that decoupled analytical expressions can be found. For instance, in [1] and [2] a decomposition of the 3D LIP model is carried out to introduce a concept called Capture point which is useful for starting and stopping phases and also for designing periodic walking gaits. Recently in 3 the 3D LIP model was used to design a biped walking pattern based on a new way of discretization named spatially quantized dynamics (SQD). In [4] the 3D LIP model is studied along with their energyoptimal gait planning based on geodesics in order to control the walking gait. As shown, the 3D LIP model is still largely used in the literature, however,

15 as it is an approximate model, the resulting walking gaits does not have the same performance when they are realized by the complete model, therefore, complementary control techniques or adjustments must be taken into account.

One of the main difficulties of walking studies is the equilibrium of the robot, i.e. to satisfy the contact hypothesis and in particular to avoid the rotation of 20 the stance foot. Thus, the constraint on the Zero Moment Point (ZMP) is crucial (see [5]). One way to ensure this constraint is satisfied is by using high-level control to impose a desired evolution for the ZMP [6].

On the other hand, a walking gait can be an "automatic" task if the floor is flat and empty, or a task precisely defined by the environment. In the first 
case, the assumption that the motion of the robot depends only on its internal states is made [7, 8, 9, 10, 11]. In the second case, the modeling must be based on a reference linked to the environment to impose a precise pose of the landing foot even in presence of perturbations. In this case, a temporal evolution of the swing foot is often prescribed [6, 12, 13, 14].

The objective of this paper is to propose a new model of the same dimension that the 3D LIP model that considers the whole dynamics of the humanoid robot, in order to develop walking gaits that deals with the issues described above, i.e. fulfilling the equilibrium condition by ensuring the stance foot is in contact with the ground, and defining the body motion as a function of internal and external variables. We have called this model: essential model. This model has been developed by taking into account the notion of zero dynamics, which is a very useful tool to analyze the internal dynamics of a system [15]. Among many applications, this tool has also been used to develop walking motions of underactuated systems such as in [16, [9], 11], 17], among others. However, unlike previous works, the original idea in this paper is to define a $3 \mathrm{D}$ dynamic relation between the two internal states (usually the horizontal position of the CoM) and the ZMP without the assumptions of the well-known LIP model. Therefore, the main contribution of this work is the development of a new model for humanoid robots that can be used instead of the 3D LIP with several 45 advantages with respect to it, as the following:

1. Unlike the 3D LIP model, the essential model is not based on a mechanical approximation (concentrated mass) of the robot but here the motion and dynamics of all the robot's body are taken into account.

2. It is possible to impose that the ZMP is in a desired location during the whole step (as shown in Cases I and II in Section 6) but also make the ZMP follows a desired path while the robot performs its motion (as shown in Case III in Section 6).

3. Impacts of the swing foot with the ground can be considered to compute periodic walking gaits (as shown in Cases II and III in Section 6). 
4. By using internal and/or external information, the desired motion of the robot can be defined. Thereby, different essential models can be developed for different purposes. For instance, the motion of the robot can be defined as a function of the position of the center of mass (CoM) in horizontal plane, thus obtaining an essential model that can be used to develop autonomous walking gaits (as shown in Case II in Section 6). On the other part, by including the time as external variable to define the essential model behavior, a precise pose of the landing foot can be imposed (as shown in Cases I and III in Section 6).

5. The proposed model can conceivably be used to develop motions in double support phases, However, in this paper, only a continuous single support (SS) phase and an instantaneous double support (DS) phase are taken into account.

On the other hand, unlike the 3D LIP model, the essential model is nonlinear, coupled and has a non-closed form formula. Despite this, many numerical studies can be conducted with this model, including gaits for climbing stairs, walking gaits on uneven ground, walking gaits that minimize energy consumption, starting or stopping steps, reaction to disturbances by means of an extension of the capture point method, among others. However, since the aim of the paper is to introduce the essential model in a more general way, these tasks are 75 not analyzed in here and they be realized in the future.

The paper is structured as follows. In order to put this proposal in context, three dynamic models used in the literature are presented in Section 2 The new model is introduced in Section 3 . Then, the connection between the essential model and the complete model in order to deduce the desired joint motions and the corresponding torques is detailed in Section 44. A brief discussion on generation of periodic walking patterns is presented in Section 5. The application of this model to the humanoid robot ROMEO as a case of study is presented in Section 6, where three cases are studied in order to show the effectiveness of the essential model. In there, a comparison with the 3D LIP model, and differ- 
ent walking gaits where impacts are considered and the ZMP follows a desired trajectory are found. Finally, the paper ends with several concluding remarks and perspectives in Section 7.

\section{The dynamic model}

The considered models of walking locomotion are inherently hybrids. They are composed of continuous differential equations which describe the motion of the robot during SS and DS phases, and discrete components where the leg transfer takes place [18. In this paper, it is assumed an instantaneous DS phase, where the change of support takes place. This assumption leads to a discontinuity in the velocities if the velocity of the swing foot is not zero at the transition.

The continuous phase

The motion of the humanoid robot during the SS phase is governed by nonlinear differential equations. In general, by considering the generalized coordinates $\boldsymbol{q} \in \mathbb{R}^{n}$ which describe the biped's configuration of dimension $n$, and the vector of velocities $\dot{\boldsymbol{q}} \in \mathbb{R}^{n}$, the dynamic model can be written as

$$
\dot{\zeta}=f_{\zeta}(\zeta)+g_{\zeta}(\zeta) u
$$

where $\boldsymbol{\zeta}=\left[\boldsymbol{q}^{\top}, \dot{\boldsymbol{q}}^{\top}\right]^{\top}$, is the state vector, $f_{\zeta}: \mathbb{R}^{2 n} \rightarrow \mathbb{R}^{2 n}, g_{\zeta}(\boldsymbol{\zeta}): \mathbb{R}^{2 n} \rightarrow$ $\mathbb{R}^{2 n \times r}$ are non linear functions and $\boldsymbol{u} \in \mathbb{R}^{r}$ is the vector of $r$ control inputs. Further details of equation (1) in the compact form obtained by the NewtonEuler algorithm are shown in Section 2.1.

Transition between support

In walking, the transition between SS phases occurs after an intermediate DS phase, which is defined when both feet are in contact with the ground. An impact of the swing foot with the ground is typically produced at the end of 
the SS phase. However in experimental tests, the impact is sometimes evaded, i.e. the swing foot touches the ground with zero velocity, in order to avoid high internal forces in the structure of the robot (such as in [14]). In any case, when an instantaneous DS is considered, the change of support must be realized when the swing foot touches the ground, namely, when the switching manifold

$$
\mathcal{S}_{\boldsymbol{q}}=\left\{\boldsymbol{q} \in \mathbb{R}^{n} \mid z_{f}(\boldsymbol{q})=0\right\}
$$

is reached, where $z_{f}(\boldsymbol{q})$ is the height of the swing foot w.r.t. the ground.

Symmetry on right and left legs is considered in this paper. Therefore, when the swing foot touches the ground a relabeling of the joint variables is performed, i.e.

$$
\boldsymbol{q}^{+}=E \boldsymbol{q}^{-}
$$

where superscripts + and - mean the instants just after and just before the impact respectively, and matrix $E$ defines the interchange of joint positions.

If an impact with the ground is considered, a change on the joint velocities occurs. Therefore, the velocities after impact can be expressed in a compact way as

$$
\dot{\boldsymbol{q}}^{+}=\Delta(\boldsymbol{q}) \dot{\boldsymbol{q}}^{-}
$$

where $\Delta(\boldsymbol{q}) \in \mathbb{R}^{n \times n}$ is a matrix that relates the joint velocities after and before the impact. Notice that if there is no impact at transition $\Delta(\boldsymbol{q})=E$.

Therefore, by taking into account the continuous phase described by (1) and the transition equations in position (3) and velocity (4), the hybrid model of the robot can be written as

$$
\left\{\begin{aligned}
\dot{\boldsymbol{\zeta}} & =f_{\zeta}(\boldsymbol{\zeta})+g_{\zeta}(\boldsymbol{\zeta}) \boldsymbol{u} & & \boldsymbol{\zeta} \notin \mathcal{S}_{\boldsymbol{q}} \\
\boldsymbol{\zeta}^{+} & =\Delta_{\zeta}\left(\boldsymbol{\zeta}^{-}\right) & & \boldsymbol{\zeta} \in \mathcal{S}_{\boldsymbol{q}}
\end{aligned}\right.
$$

where $\Delta_{\zeta}(\boldsymbol{\zeta})=\left[\left(E \boldsymbol{q}^{-}\right)^{\top},\left(\Delta(\boldsymbol{q}) \dot{\boldsymbol{q}}^{-}\right)^{\top}\right]^{\top}$. 


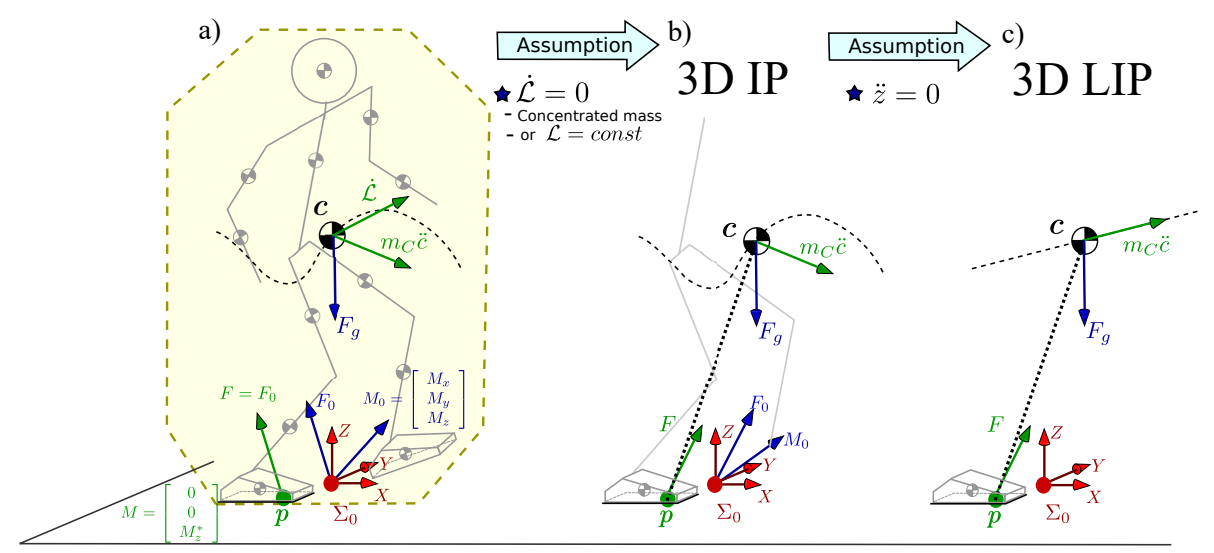

Figure 1: a) Centroidal model. b) Inverted Pendulum model c) Linear Inverted Pendulum model. The gravity force $\boldsymbol{F}_{g}$ acts on the CoM as an external force. The inertial force and inertial moment are denoted as $m_{c} \ddot{\boldsymbol{c}}$ and $\dot{\mathcal{L}}$ respectively. The ground reaction wrench $\boldsymbol{F}_{0}$ and $\boldsymbol{M}_{0}$ are expressed in a reference frame $\Sigma_{0}$. This frame can be placed anywhere. However, if the reaction wrench is expressed on the CoP (point $\boldsymbol{p}$ ) it can be formed instead by the reduced vector $\boldsymbol{F}$ and $\boldsymbol{M}$.

In the remaining of this section a brief explanation of the three dynamic models most used in literature to model the continuous phase in SS phase of humanoid robots is carried out in order to set the stages for our proposal: The essential model.

\subsection{Case 1: The complete dynamic model}

The dynamic model which describes the motion of a humanoid robot in the SS phase can be written as

$$
A(\boldsymbol{q}) \ddot{\boldsymbol{q}}+\boldsymbol{d}(\boldsymbol{q}, \dot{\boldsymbol{q}})=B \boldsymbol{\tau},
$$

where $\boldsymbol{q}, \dot{\boldsymbol{q}}, \ddot{\boldsymbol{q}} \in \mathbb{R}^{n}$ are the position, velocities, and accelerations of the joints, respectively, $A \in \mathbb{R}^{n \times n}$ is the inertia matrix, $\boldsymbol{d} \in \mathbb{R}^{n}$ is the vector of centrifugal, Coriolis and gravitational forces, $B \in \mathbb{R}^{n \times r}$ is the input matrix] (a study of

\footnotetext{
${ }^{1}$ If $n>r$ an underactuated robot is considered. This condition is common when a pointedfeet robot is studied or during the phase where the robot is supported on its foot tip. If $n=r$ a complete actuated robot is considered, namely a robot with feet and flat foot contact.
} 
matrix $B$ for the planar case is found in [19]) and $\tau \in \mathbb{R}^{r}$ is the vector of applied torques by actuators at joints.

The dynamic model (6) considers the inertial frame $\Sigma_{0}$ linked to the stance foot (see Fig1(a)). Therefore, since parametrization with implicit contact is used the ground reaction force does not appear. Moreover, by considering $B=I$ for the particular choice of actuated coordinates when $r=n$, the dynamic equation (6) and the ground reaction wrench $\left[\boldsymbol{F}_{0}^{\top}, \boldsymbol{M}_{0}^{\top}\right]^{\top}$ at the inertial frame $\Sigma_{0}$ can be computed with the Newton-Euler algorithm, namely

$$
\left[\begin{array}{c}
\boldsymbol{F}_{0} \\
\boldsymbol{M}_{0} \\
\boldsymbol{\tau}
\end{array}\right]=\boldsymbol{N E}(\boldsymbol{q}, \dot{\boldsymbol{q}}, \ddot{\boldsymbol{q}})
$$

where $\boldsymbol{F}_{0}=\left[F_{x}, F_{y}, F_{z}\right]^{\top}$ and $\boldsymbol{M}_{0}=\left[\begin{array}{lll}M_{x}, & M_{y}, M_{z}\end{array}\right]^{\top}$. Furthermore, this equation is linear in the acceleration $\ddot{\boldsymbol{q}}$, i.e.

$$
\left[\begin{array}{c}
\boldsymbol{F}_{0} \\
\boldsymbol{M}_{0} \\
\boldsymbol{\tau}
\end{array}\right]=\left[\begin{array}{c}
A_{F}(\boldsymbol{q}) \\
A_{M}(\boldsymbol{q}) \\
A(\boldsymbol{q})
\end{array}\right] \ddot{\boldsymbol{q}}+\left[\begin{array}{c}
\boldsymbol{d}_{F}(\boldsymbol{q}, \dot{\boldsymbol{q}}) \\
\boldsymbol{d}_{M}(\boldsymbol{q}, \dot{\boldsymbol{q}}) \\
\boldsymbol{d}(\boldsymbol{q}, \dot{\boldsymbol{q}})
\end{array}\right]
$$

where $A_{F} \in \mathbb{R}^{3 \times n}, A_{M} \in \mathbb{R}^{3 \times n}, \boldsymbol{d}_{F} \in \mathbb{R}^{3}$ and $\boldsymbol{d}_{M} \in \mathbb{R}^{3}$ are matrices and vectors used to compute the reaction wrench. Thus, equation (8) can be arranged as

$$
\left[\begin{array}{c}
\boldsymbol{F}_{0} \\
\boldsymbol{M}_{0} \\
\boldsymbol{\tau}
\end{array}\right]=A_{e}(\boldsymbol{q}) \ddot{\boldsymbol{q}}+\boldsymbol{d}_{e}(\boldsymbol{q}, \dot{\boldsymbol{q}}),
$$

where $A_{e}=\left[A_{F}^{\top}, A_{M}^{\top}, A^{\top}\right]^{\top} \in \mathbb{R}^{n+6 \times n}$ and $\boldsymbol{d}_{e}=\left[\boldsymbol{d}_{F}^{\top}, \boldsymbol{d}_{M}^{\top}, \boldsymbol{d}^{\top}\right]^{\top} \in \mathbb{R}^{n+6}$.

\subsection{Case 2: The Centroidal model}

The dynamic model that highlights the difficulty of walking is the equilibrium of the complete robot, also called the Centroidal model [20] (see Fig. 1(a)). In here, the position of the CoM w.r.t. the frame $\Sigma_{0}$ is denoted by $\boldsymbol{c}=[x, y, z]^{\top}$, and the mass of the robot is denoted by $m_{c}$. The linear acceleration of the CoM 
is $\ddot{\boldsymbol{c}}$ and its angular momentum around its CoM is denoted as $\mathcal{L}^{2}$ In this model,

\footnotetext{
${ }^{2}$ For a multi-body system, the angular momentum around its CoM is given by $\mathcal{L}=$
$\sum_{i=1}^{N}\left[I_{i} \omega_{i}+m_{i}\left(\boldsymbol{c}-\boldsymbol{c}_{i}\right) \times \boldsymbol{v}_{i}\right]$, where $N$ is the number of bodies of the robot, $I_{i}$ is the in-

${ }^{2}$ For a multi-body system, the angular momentum around its CoM is given by $\mathcal{L}=$
$\sum_{i=1}^{N}\left[I_{i} \omega_{i}+m_{i}\left(\boldsymbol{c}-\boldsymbol{c}_{i}\right) \times \boldsymbol{v}_{i}\right]$, where $N$ is the number of bodies of the robot, $I_{i}$ is the inertia tensor at the $\mathrm{CoM}_{i}, \omega_{i}$ is the angular velocity and $\boldsymbol{c}_{i}$ and $\boldsymbol{v}_{i}$ are the position and velocity of the $\mathrm{CoM}_{i}$
} 
The last condition is the more constraining, therefore it is important to know the position of the CoP during all the walking gait. This position can be easily obtained from equations 10 and $(11)$ as

$$
\begin{aligned}
& p_{x}=x-\frac{z \ddot{x}}{\ddot{z}+g}-\frac{\dot{\mathcal{L}}_{y}}{m_{c} \ddot{z}+m_{c} g} \\
& p_{y}=y-\frac{z \ddot{y}}{\ddot{z}+g}+\frac{\dot{\mathcal{L}}_{x}}{m_{c} \ddot{z}+m_{c} g} .
\end{aligned}
$$

On the other hand, the position of the ZMP can be computed based on the ground reaction wrench at frame $\Sigma_{0}$, namely

$$
\boldsymbol{M}_{0}-\boldsymbol{p} \times \boldsymbol{F}_{0}=\boldsymbol{M}
$$

By considering the inertial frame $\Sigma_{0}$ on a flat ground, $p_{z}=0$. Then, the horizontal position of $\mathrm{CoP}$ is obtained from the two first rows of equation (14), i.e.

$$
\begin{aligned}
& p_{x}=\frac{-M_{y}}{F_{z}} \\
& p_{y}=\frac{M_{x}}{F_{z}} .
\end{aligned}
$$

The position $\left(p_{x}, p_{y}\right)$ must be always inside the convex hull of support for all time in order to equations 12 and 13 be valid.

\subsection{Case 3: 3D LIP model}

The equations 12 and 13 can be used to build a model that contains the main properties of walking. By making the assumptions that the vertical acceleration of the $\mathrm{CoM}$ and the derivative w.r.t. time of the angular momentum are zero, the 3D LIP model arises, as shown in Fig. 1.(c) (notice that the motion is restricted to a plane not necessary horizontal [21]). The last assumption implies two possibilities: 1) the total mass of the robot is constrained in one point; 2) the total angular momentum around the CoM is constant (this choice could restrict the motion of the CoM, that is why it is not often used). Thus, 
by using these assumptions in equations $\sqrt{12}$ and $(13)$ we have

$$
\begin{aligned}
& p_{x}=x-\frac{z \ddot{x}}{g}, \\
& p_{y}=y-\frac{z \ddot{y}}{g} .
\end{aligned}
$$

From these equations, the well-known 3D LIP model proposed by Kajita [21] is obtained, which is given by

$$
\begin{aligned}
\ddot{x} & =\frac{g}{z}\left(x-p_{x}\right), \\
\ddot{y} & =\frac{g}{z}\left(y-p_{y}\right) .
\end{aligned}
$$

The 3D LIP model is often used to study walking gaits due to the fact it captures some essential properties of walking, such as the limit of the ZMP and the effect of gravity. This model is composed of two linear differential equations of second order that allow to define the evolution of the CoM when the position of the $\mathrm{CoP}$ is known. Moreover, its dynamics in the sagittal plane (defined by the axis of walking direction $X$ ) is decoupled from those in the frontal plane (defined along $Y$ axis).

In practice, after the calculation of the evolution of the CoM by using the 3D LIP model, a desired motion of a massless swing leg and upper body are often added in order to define joint motions. However, since the evolution of the ZMP is affected by the joint motions in the complete model, the motions of the real swing leg and upper body need to be considered from the beginning of walking gait developments. In the next section, a reduced model that takes into account the motions of the real leg and upper body from the beginning is developed.

\section{The proposed essential model}

A new model for generation of walking gaits for humanoid robots called essential model is proposed. A fully actuated $n$-DOF robot is considered. Notice that in SS phase, due to the limitations of the unilateral force in the stance foot, a very restricted control of the CoM can be achieved due to the limited 
ankle torque and the fact that the ZMP must be inside of the support polygon. Therefore, in order to produce a model which is close to the 3D LIP model, the development of the essential model considers to control the position of the ZMP but not the position of the CoM along the axis $\mathrm{x}$ and $\mathrm{y}$ and their time derivatives. Therefore, the proposed model will be composed of two nonlinear differential equations of second order. This model is not based on approximation, but on the hypothesis that the joints are coordinated based on some internal and/or external information. A particular case is when the motion of the robot is expressed as a function of the position of the CoM, while another is when it is expressed as a function of time. By considering the last case, in this paper the essential model is compared with the 3D LIP model with better performance. Furthermore, this essential model can be used to generate walking gaits that 225 consider impacts with the ground and still keep the ZMP in a desired location as will be shown in further sections.

\subsection{Development of the Essential Model}

The aim of the essential model is to describe the evolution of the noncontrolled (or free) internal state $\boldsymbol{q}_{f} \in \mathbb{R}^{2}$ as a function of the desired position of the ZMP, similar to the 3D LIP model where the dynamics of the CoM is obtained as a function of the CoP, as shown in Fig. 1(c) and equations 19 and (20). For the development of this proposal a fully actuated robot is considered. However, a pointed-feet contact model can be also considered since the position of the ZMP can be imposed.

In order to develop this model, a general biped robot as the one shown in Fig. 1(a) is considered. Thus, the equilibrium equations 15 and 16 given by the Centroidal model, must be satisfied for the desired ZMP positions $p_{x, d}$ and $p_{y, d}$ i.e.

$$
\begin{aligned}
& p_{x, d} F_{z}+M_{y}=0 \\
& p_{y, d} F_{z}-M_{x}=0 .
\end{aligned}
$$


Subsequently, it is desired that the robot's joints perform a particular evolution such that equations (21) and $(22)$ be fulfilled for all the time. In order to achieve that, it is proposed (by assuming perfect tracking of the reference trajectories) that the evolution of the joint vector $\boldsymbol{q}$ performs a non-trivial desired trajectory $\boldsymbol{q}_{d}$ based on internal and possible external states, i.e.

$$
\boldsymbol{q}=\boldsymbol{q}_{d}(\boldsymbol{\vartheta})
$$

where $\boldsymbol{q} \in \mathbb{R}^{n}$ is the joint vector, $\boldsymbol{q}_{d} \in \mathbb{R}^{n}$ is the desired joint vector obtained from inverse kinematics (see Section 4 , $\boldsymbol{\vartheta}=\left[\boldsymbol{q}_{f}^{\top}, \boldsymbol{\phi}^{\top}\right]^{\top} \in \mathbb{R}^{m+2}, \boldsymbol{q}_{f} \in \mathbb{R}^{2}$ is the not-controlled internal variable vector and $\phi \in \mathbb{R}^{m}$ is a possible external variable vector with $m \geq 0$. In this paper, the horizontal position $x$ and $y$ of the $\mathrm{CoM}$ are chosen as internal variables, i.e. $\boldsymbol{q}_{f}=[x, y]^{\top}{ }^{3}$. Therefore, in here, it is desired to obtain the dynamics of the CoM by imposing the ZMP location. Let us remark that if the external variable vector $\phi$ is used for modifying the evolution of the joints, the derivatives w.r.t. time, i.e. $\dot{\phi}$ and $\ddot{\phi}$ must be known.

By assuming equation (23) is fulfilled for all time, i.e. by neglecting tracking error in the control, its first and second derivative w.r.t. time are also fulfilled, 250 i.e.

$$
\dot{\boldsymbol{q}}=J_{\vartheta}(\boldsymbol{\vartheta}) \dot{\vartheta}
$$

and

$$
\ddot{\boldsymbol{q}}=J_{\vartheta}(\boldsymbol{\vartheta}) \ddot{\boldsymbol{\vartheta}}+\dot{J}_{\vartheta}(\boldsymbol{\vartheta}, \dot{\boldsymbol{\vartheta}}) \dot{\boldsymbol{\vartheta}}
$$

where $J_{\vartheta}(\boldsymbol{\vartheta})=\frac{\partial \boldsymbol{q}_{d}(\boldsymbol{\vartheta})}{\partial \boldsymbol{\vartheta}} \in \mathbb{R}^{n \times m+2}$.

Now, in order to compute the ground reaction force and moment due to the proposed joint trajectories, the Newton-Euler algorithm is used. Thus, by

\footnotetext{
${ }^{3}$ However, it is conceivable that other coordinates that describe the evolution of the robot in the frontal and sagittal planes can be used as internal variables, such as the horizontal evolution of the hip or the joint evolution of the support ankle (i.e. $q_{1}$ and $q_{2}$ in Figure 4, etc.
} 
255

substituting (25) into (9) we have

$$
\boldsymbol{N} \boldsymbol{E}=A_{e}\left[J_{\vartheta} \ddot{\boldsymbol{\vartheta}}+\dot{J}_{\vartheta} \dot{\boldsymbol{\vartheta}}\right]+\boldsymbol{d}_{e}(\vartheta, \dot{\boldsymbol{\vartheta}})
$$

where $\boldsymbol{N} \boldsymbol{E}=\left[\boldsymbol{F}_{0}, \boldsymbol{M}_{0}, \boldsymbol{\tau}\right]^{\top}$, and then

$$
\boldsymbol{N} \boldsymbol{E}=A_{\vartheta} \ddot{\vartheta}+\boldsymbol{d}_{\vartheta}
$$

where $A_{\vartheta}(\boldsymbol{\vartheta}) \in \mathbb{R}^{n+6 \times m+2}$ and $\boldsymbol{d}_{\vartheta}(\boldsymbol{\vartheta}, \dot{\boldsymbol{\vartheta}}) \in \mathbb{R}^{n+6}$ are given by

$$
\begin{aligned}
A_{\vartheta} & =A_{e} J_{\vartheta} \\
\boldsymbol{d}_{\vartheta} & =A_{e} \dot{J}_{\vartheta} \dot{\boldsymbol{\vartheta}}+\boldsymbol{d}_{e} .
\end{aligned}
$$

A numerical procedure for computing matrix $A_{\vartheta}$ and vector $\boldsymbol{d}_{\vartheta}$ is shown in Appendix.

Equation 27 represents the reduced system as a function of $\boldsymbol{\vartheta}$ by assuming the reference trajectories 23 are fulfilled. This equation can be split into

$$
\boldsymbol{N} \boldsymbol{E}=A_{\vartheta}(:, 1) \ddot{x}+A_{\vartheta}(:, 2) \ddot{y}+A_{\vartheta}(:, 3) \ddot{\phi}_{1}+\ldots+A_{\vartheta}(:, m) \ddot{\phi}_{m}+\boldsymbol{d}_{\vartheta}
$$

By taking into account rows 3 to 5 of equation 28 we have that

$$
\begin{aligned}
& F_{z}=A_{\vartheta}(3,1) \ddot{x}+A_{\vartheta}(3,2) \ddot{y}+A_{\vartheta}(3,3) \ddot{\phi}_{1}+\ldots+A_{\vartheta}(3, m) \ddot{\phi}_{m}+\boldsymbol{d}_{\vartheta}(3) \\
& M_{x}=A_{\vartheta}(4,1) \ddot{x}+A_{\vartheta}(4,2) \ddot{y}+A_{\vartheta}(4,3) \ddot{\phi}_{1}+\ldots+A_{\vartheta}(4, m) \ddot{\phi}_{m}+\boldsymbol{d}_{\vartheta}(4) \\
& M_{y}=A_{\vartheta}(5,1) \ddot{x}+A_{\vartheta}(5,2) \ddot{y}+A_{\vartheta}(5,3) \ddot{\phi}_{1}+\ldots+A_{\vartheta}(5, m) \ddot{\phi}_{m}+\boldsymbol{d}_{\vartheta}(5)
\end{aligned}
$$

In order to obtain the desired position of the ZMP, equations (21) and (22) must be satisfied. Then, by using 29 into 21 and 22 we get

$$
\begin{aligned}
& p_{x, d}\left[A_{\vartheta}(3,1) \ddot{x}+A_{\vartheta}(3,2) \ddot{y}+A_{\vartheta}(3,3) \ddot{\phi}_{1}+\cdots+A_{\vartheta}(3, m) \ddot{\phi}_{m}+\boldsymbol{d}_{\vartheta}(3)\right]+ \\
& {\left[A_{\vartheta}(5,1) \ddot{x}+A_{\vartheta}(5,2) \ddot{y}+A_{\vartheta}(5,3) \ddot{\phi}_{1}+\cdots+A_{\vartheta}(5, m) \ddot{\phi}_{m}+\boldsymbol{d}_{\vartheta}(5)\right]=0} \\
& p_{y, d}\left[A_{\vartheta}(3,1) \ddot{x}+A_{\vartheta}(3,2) \ddot{y}+A_{\vartheta}(3,3) \ddot{\phi}_{1}+\cdots+A_{\vartheta}(3, m) \ddot{\phi}_{m}+\boldsymbol{d}_{\vartheta}(3)\right]- \\
& {\left[A_{\vartheta}(4,1) \ddot{x}+A_{\vartheta}(4,2) \ddot{y}+A_{\vartheta}(4,3) \ddot{\phi}_{1}+\cdots+A_{\vartheta}(4, m) \ddot{\phi}_{m}+\boldsymbol{d}_{\vartheta}(4)\right]=0 .}
\end{aligned}
$$

Equations 30 and 31 can be arranged by splitting the internal states and external variables, such as

$$
A_{f}(\boldsymbol{\vartheta}, \boldsymbol{p}) \ddot{\boldsymbol{q}}_{f}+A_{\phi}(\boldsymbol{\vartheta}, \boldsymbol{p}) \ddot{\boldsymbol{\phi}}+\boldsymbol{d}_{R}(\boldsymbol{\vartheta}, \dot{\boldsymbol{\vartheta}})=0
$$




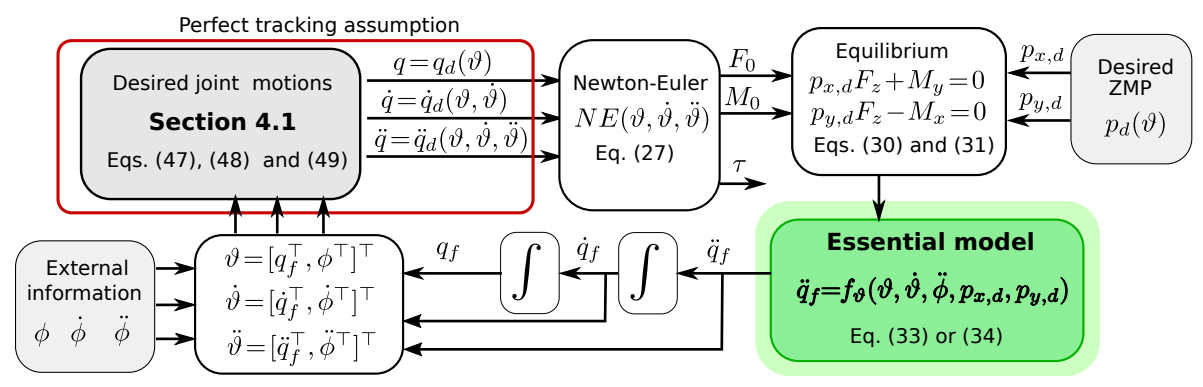

Figure 2: A summary of the development of the essential model.

where $A_{f}(\boldsymbol{\vartheta}, \boldsymbol{p}) \in \mathbb{R}^{2 \times 2}, A_{\phi}(\boldsymbol{\vartheta}, \boldsymbol{p}) \in \mathbb{R}^{2 \times m}$ and $\boldsymbol{d}_{R}(\boldsymbol{\vartheta}, \dot{\boldsymbol{\vartheta}}) \in \mathbb{R}^{2}$ are given by

$$
\begin{aligned}
A_{f} & =\left[\begin{array}{lll}
p_{x, d} A_{\vartheta}(3,1)+A_{\vartheta}(5,1) & p_{x, d} A_{\vartheta}(3,2)+A_{\vartheta}(5,2) \\
p_{y, d} A_{\vartheta}(3,1)+A_{\vartheta}(4,1) & p_{y, d} A_{\vartheta}(3,2)+A_{\vartheta}(4,2)
\end{array}\right] \\
A_{\phi} & =\left[\begin{array}{lll}
p_{x, d} A_{\vartheta}(3,3)+A_{\vartheta}(5,3) & \cdots & p_{x, d} A_{\vartheta}(3, m)+A_{\vartheta}(5, m) \\
p_{y, d} A_{\vartheta}(3,3)+A_{\vartheta}(4,3) & \cdots & p_{y, d} A_{\vartheta}(3, m)+A_{\vartheta}(4, m)
\end{array}\right] \\
\boldsymbol{d}_{R}= & {\left[\begin{array}{l}
p_{x, d} \boldsymbol{d}_{\vartheta}(3)+\boldsymbol{d}_{\vartheta}(5) \\
p_{y, d} \boldsymbol{d}_{\vartheta}(3)+\boldsymbol{d}_{\vartheta}(4)
\end{array}\right] . }
\end{aligned}
$$

Finally, due to the good choice of $\boldsymbol{q}_{f}$, matrix $A_{f}$ is invertible. Therefore,

from equation 32 the essential model is computed as

$$
\ddot{\boldsymbol{q}}_{f}=-A_{f}^{-1}\left(A_{\phi} \ddot{\boldsymbol{\phi}}+\boldsymbol{d}_{R}\right)
$$

or well

$$
\left[\begin{array}{l}
\ddot{x} \\
\ddot{y}
\end{array}\right]=\boldsymbol{f}_{\vartheta}\left(\boldsymbol{\vartheta}, \dot{\boldsymbol{\vartheta}}, \ddot{\boldsymbol{\phi}}, p_{x, d}, p_{y, d}\right) .
$$

In this way, the dynamic behavior of the humanoid robot that keeps the ZMP in a desired position $\left(p_{x, d}, p_{y, d}\right)$ during all the gait is described by the essential model (34). A summary of this procedure is shown in Fig. 2 Note that in the essential model, the simplification of the model is not based on any approximation of the dynamics, but on the assumption that the control is such that the reference trajectories of the joints are perfectly tracked. This model can be designed as a function of internal states and possible external 
variables. One simple case of the essential model is by developing it only as a

function of the horizontal position of the CoM as internal states. Nevertheless, if a desired behaviour is imposed by external information, the motion of the joint can be expressed as a function of other variables and the dimension of the system will be the same. In the following, the most obvious cases of the application of this essential model will be shown. Namely, the cases when there are no external information, i.e. without considering $\phi$, and when the time is included as external information, i.e. $\phi=t$. Let us remark that not only time but other kind of external variables can be used as external information, such as the CoM of another biped robot, the CoM of a human, etc. However, this study is out of the scope of this paper but it is expected to be performed in the future.

\subsection{Essential model based only on its CoM}

The hypothesis for the development of this model is the assumption that the motion of the joints are defined as a function of the internal information $\boldsymbol{q}_{f}$, i.e $\boldsymbol{\vartheta}=\boldsymbol{q}_{f}$. Thus, since $\boldsymbol{\phi}$ is not considered the term $A_{\phi} \ddot{\boldsymbol{\phi}}$ on the essential model (33) vanishes and the model is reduced to

$$
\left[\begin{array}{c}
\ddot{x} \\
\ddot{y}
\end{array}\right]=-A_{f}^{-1} \boldsymbol{d}_{R}=\boldsymbol{f}_{x, y}\left(x, y, \dot{x}, \dot{y}, p_{x, d}, p_{y, d}\right)
$$

This particular model can be used to perform walking gaits by making the robot walks only by considering its state. This description of the gait is based on virtual constraints and it has been efficiently used in numerous studies such as [7, 8, 9, 10, 11, among others. Notice that, trajectories based on the internal states of the robot are more natural since the robot will not try to catch a time varying function after some perturbation. Nevertheless, the desired step time lapse is not ensured.

\subsection{Essential model based on its CoM and time}

By including the time as external state, the motion of the robot during a step time $T$ at each step is controlled. The hypothesis for the development 
of this model is the assumption that the motion of the joints are defined as a function of the internal states $\boldsymbol{q}_{f}$ and $\boldsymbol{\phi}=t$ as external state. Notice that by only including the time, $\dot{\phi}=1$ and $\ddot{\phi}=0$. Thus as the previous case, term $A_{\phi} \ddot{\phi}$ on the essential model 33 vanishes and the model is reduced to

$$
\left[\begin{array}{l}
\ddot{x} \\
\ddot{y}
\end{array}\right]=-A_{f}^{-1} \boldsymbol{d}_{R}=\boldsymbol{f}_{x, y, t}\left(t, x, y, \dot{x}, \dot{y}, p_{x, d}, p_{y, d}\right)
$$
Thus, vector $\boldsymbol{\xi}$ will be composed of the controlled variables $\boldsymbol{q}_{c}(\boldsymbol{q})$ and the two internal non-controlled variables $\boldsymbol{q}_{f}$, i.e.

$$
\boldsymbol{\xi}=\boldsymbol{f}(\boldsymbol{q})=\left[\begin{array}{c}
\boldsymbol{q}_{c}(\boldsymbol{q}) \\
\boldsymbol{q}_{f}(\boldsymbol{q})
\end{array}\right]=\left[\begin{array}{c}
\boldsymbol{q}_{c}(\boldsymbol{q}) \\
x(\boldsymbol{q}) \\
y(\boldsymbol{q})
\end{array}\right] .
$$


On the other hand, a desired trajectory for the $n-2$ controlled variables 325 is defined as a function of the generalized vector $\boldsymbol{\vartheta}=\left[x, y, \phi_{1}, \ldots, \phi_{m}\right]^{\top}$, i.e. $\boldsymbol{q}_{c}^{d}(\boldsymbol{\vartheta})$. When trajectories $\boldsymbol{q}_{c}^{d}(\boldsymbol{\vartheta})$ are defined, the output vector is created

$$
\boldsymbol{y}=\boldsymbol{q}_{c}(\boldsymbol{q})-\boldsymbol{q}_{c}^{d}(\boldsymbol{\vartheta})
$$

The choice of the desired trajectories $\boldsymbol{q}_{c}^{d}(\boldsymbol{\vartheta})$ may be not obvious. However, in here, the assumption of well-defined trajectories is made. Let us call $\boldsymbol{\xi}_{d}$ the vector of desired generalized variables defined by

$$
\boldsymbol{\xi}_{d}=\boldsymbol{f}_{d}(\boldsymbol{\vartheta})=\left[\begin{array}{c}
\boldsymbol{q}_{c}^{d}(\boldsymbol{\vartheta}) \\
\boldsymbol{q}_{f}
\end{array}\right]=\left[\begin{array}{c}
\boldsymbol{q}_{c}^{d}(\boldsymbol{\vartheta}) \\
x \\
y
\end{array}\right]
$$

330

When the system achieves the desired trajectory $\boldsymbol{q}_{c}^{d}(\boldsymbol{\vartheta})$, i.e. $\boldsymbol{y} \equiv \mathbf{0}$ the internal dynamics due to the non-controlled variables is the only dynamics that remains and it is said the system evolves in the zero dynamics [15]. Therefore, recalling that the simplification of the essential model is based on the assumption of a perfect tracking, equation (37) could be replaced by (39). Then, since $\boldsymbol{\xi} \equiv \boldsymbol{\xi}_{d}$, the position of the controlled variables $\boldsymbol{q}_{c}(\boldsymbol{q})$ can be deduced by just knowing the states $\boldsymbol{\vartheta}$. Furthermore, joint positions, velocities and accelerations (i.e. $\boldsymbol{q}, \dot{\boldsymbol{q}}$ and $\ddot{\boldsymbol{q}}$ ) that allow to follow the desired trajectory $\boldsymbol{q}_{c}^{d}(\boldsymbol{\vartheta})$ can be also deduced as shown below.

\subsection{The joint motion}

In order to compute the joint motions, the first and second derivative w.r.t. time of equation 37 is needed, i.e

$$
\dot{\xi}=J_{\xi}(\boldsymbol{q}) \dot{\boldsymbol{q}}
$$

and

$$
\ddot{\boldsymbol{\xi}}=J_{\xi}(\boldsymbol{q}) \ddot{\boldsymbol{q}}+\dot{J}_{\xi}(\boldsymbol{q}) \dot{\boldsymbol{q}}
$$

where $J_{\xi}(\boldsymbol{q})=\frac{\partial \boldsymbol{f}(\boldsymbol{q})}{\partial \boldsymbol{q}} \in \mathbb{R}^{n \times n}$. 
On the other hand, from the first and second derivative w.r.t. time of equa-

$$
\dot{\boldsymbol{\xi}}_{d}=\frac{\partial \boldsymbol{f}_{d}(\boldsymbol{\vartheta})}{\partial \boldsymbol{\vartheta}} \dot{\boldsymbol{\vartheta}}=J_{d}(\boldsymbol{\vartheta}) \dot{\boldsymbol{\vartheta}}
$$

and

$$
\ddot{\boldsymbol{\xi}}_{d}=J_{d}(\boldsymbol{\vartheta}) \ddot{\boldsymbol{\vartheta}}+\dot{J}_{d}(\boldsymbol{\vartheta}) \dot{\boldsymbol{\vartheta}}
$$

where $J_{d}(\boldsymbol{\vartheta}) \in \mathbb{R}^{n \times m+2}$ is defined as

$$
J_{d}(\boldsymbol{\vartheta})=\left[\begin{array}{ccccc}
\frac{\partial \boldsymbol{q}_{c}^{d}(\boldsymbol{\vartheta})}{\partial x} & \frac{\partial \boldsymbol{q}_{c}^{d}(\boldsymbol{\vartheta})}{\partial y} & \frac{\partial \boldsymbol{q}_{c}^{d}(\boldsymbol{\vartheta})}{\partial \phi_{1}} & \ldots & \frac{\partial \boldsymbol{q}_{c}^{d}(\boldsymbol{\vartheta})}{\partial \phi_{m}} \\
1 & 0 & 0 & \ldots & 0 \\
0 & 1 & 0 & \ldots & 0
\end{array}\right]
$$

It is assumed that $\boldsymbol{y} \equiv \mathbf{0}, \dot{\boldsymbol{y}} \equiv \mathbf{0}$ and $\ddot{\boldsymbol{y}} \equiv \mathbf{0}$. So, by relating equations (37) and (39), 40 and 42, and (41) and 43 we have

$$
\begin{gathered}
\boldsymbol{f}(\boldsymbol{q})=\boldsymbol{f}_{d}(\boldsymbol{\vartheta}) \\
J_{\xi}\left(\boldsymbol{q}_{d}(\boldsymbol{\vartheta})\right) \dot{\boldsymbol{q}}=J_{d}(\boldsymbol{\vartheta}) \dot{\boldsymbol{\vartheta}} \\
J_{\xi}\left(\boldsymbol{q}_{d}(\boldsymbol{\vartheta})\right) \ddot{\boldsymbol{q}}+\dot{J}_{\xi}\left(\boldsymbol{q}_{d}(\boldsymbol{\vartheta})\right) \dot{\boldsymbol{q}}=J_{d}(\boldsymbol{\vartheta}) \ddot{\boldsymbol{\vartheta}}+\dot{J}_{d}(\boldsymbol{\vartheta}) \dot{\boldsymbol{\vartheta}}
\end{gathered}
$$

Thus, joint variables $\boldsymbol{q}, \dot{\boldsymbol{q}}$ and $\ddot{\boldsymbol{q}}$ can be deduced from the state $\boldsymbol{\vartheta}$ and its derivatives w.r.t. time $\dot{\boldsymbol{\vartheta}}$ and $\ddot{\vartheta}$, as

$$
\begin{aligned}
& \boldsymbol{q}=\boldsymbol{f}^{-1}\left(\boldsymbol{f}_{d}(\boldsymbol{\vartheta})\right) \\
& \dot{\boldsymbol{q}}=J_{\xi}^{-1}(\boldsymbol{\vartheta}) J_{d}(\boldsymbol{\vartheta}) \dot{\boldsymbol{\vartheta}} \\
& \ddot{\boldsymbol{q}}=J_{\xi}^{-1}(\boldsymbol{\vartheta})\left[J_{d}(\boldsymbol{\vartheta}) \ddot{\boldsymbol{\vartheta}}+\dot{J}_{d}(\boldsymbol{\vartheta}, \dot{\boldsymbol{\vartheta}}) \dot{\boldsymbol{\vartheta}}-\dot{J}_{\xi}(\boldsymbol{\vartheta}, \dot{\boldsymbol{\vartheta}}) J_{\xi}^{-1}(\boldsymbol{\vartheta}) J_{d}(\boldsymbol{\vartheta}) \dot{\boldsymbol{\vartheta}}\right]
\end{aligned}
$$

where $J_{\xi}$ is invertible by assuming a good choice of $\boldsymbol{q}_{c}^{d}$, and 49 comes from solving $\ddot{\boldsymbol{q}}$ in 46 and using 48 . Finally, by defining

$$
J_{\vartheta}(\boldsymbol{\vartheta})=J_{\xi}^{-1}(\boldsymbol{\vartheta}) J_{d}(\boldsymbol{\vartheta})
$$

equations (24) and (25) are recovered and the essential model can be computed.

The term $\dot{J}_{\vartheta} \dot{\vartheta}$ in 25 is directly obtained from the last two terms of equation 49 , i.e.

$$
\dot{J}_{\vartheta} \dot{\boldsymbol{\vartheta}}=J_{\xi}^{-1} \dot{J}_{d} \dot{\boldsymbol{\vartheta}}-J_{\xi}^{-1} \dot{J}_{\xi} J_{\xi}^{-1} J_{d} \dot{\boldsymbol{\vartheta}}
$$


$350 \quad$ Notice that $-J_{\xi}^{-1} \dot{J}_{\xi} J_{\xi}^{-1}=\frac{\mathrm{d}}{\mathrm{d} t}\left(J_{\xi}^{-1}\right){ }^{4}$

Let us remark that joint positions are not directly obtained from equation (47), but they are computed by finding the numerical solution of $\boldsymbol{\xi}_{d}-\boldsymbol{\xi}$ by means of the Newton-Raphson method.

\subsection{The joint torques}

Once the joint positions, velocities and accelerations are computed the joint torques in SS phase can be obtained by using the NE algorithm (7). In DS phase, the distribution of forces between the feet must be taken into account. However this analysis is not carried out in here.

\section{Generation of periodic walking patterns}

In this section, the generation of periodic walking patterns are studied. The studied gaits are composed of a SS phase and an instantaneous DS phase. The starting and ending phases can also be studied but they are out of the scope of this paper.

\subsection{The evolution of the CoM}

Due to the mass distribution, and to the eventual loss of angular momentum at impact, a periodic motion of the CoM by considering the complete model is not necessarily a symmetric motion along the sagittal and frontal planes [8], as in the case of the 3D LIP model. Therefore, the periodic motion characterised by the state of the CoM at the end of a step is defined as

$$
\boldsymbol{x}^{-*}=\left[x^{-*}, y^{-*}, \dot{x}^{-*}, \dot{y}^{-*}\right]^{\top}
$$

with

$$
\begin{aligned}
& x^{-*}=\frac{S}{2}+D_{x}, \\
& y^{-*}=\frac{D}{2}+D_{y},
\end{aligned}
$$

\footnotetext{
${ }^{4}$ From the fact that $\frac{\mathrm{d}}{\mathrm{d} t}\left(J_{\xi} J_{\xi}^{-1}\right)=0_{n \times n}$, it can be expanded it as $\dot{J}_{\xi} J_{\xi}^{-1}+J_{\xi} \frac{\mathrm{d}}{\mathrm{d} t}\left(J_{\xi}^{-1}\right)=0$ and the equivalence is obtained.
} 

placements of the CoM in $X$ and $Y$ directions.

By using the transition model (3) and (4) the initial states of the CoM at the beginning of a periodic step $\boldsymbol{x}^{+*}\left(\boldsymbol{q}^{+}, \dot{\boldsymbol{q}}^{+}\right)=\left[x^{+*}, y^{+*}, \dot{x}^{+*}, \dot{y}^{+*}\right]^{\top}$ are deduced. During the transition, the configuration of the robot is constant but the stance leg changes, therefore the initial position of the CoM w.r.t the new stance leg is given by

$$
\begin{aligned}
& x^{+*}=-\frac{S}{2}+D_{x}, \\
& y^{+*}=\frac{D}{2}-D_{y} .
\end{aligned}
$$

Notice that, without impact, the velocities of the CoM before and after the transition are the same (by taking into account the change of the frame in $Y$ direction), i.e. $\dot{x}^{+*}=\dot{x}^{-*} \dot{y}^{+*}=-\dot{y}^{-*}$. In the simulations the periodic state is obtained numerically such that after one cycle (i.e. an instantaneous DS phase plus a SS phase) the same state is obtained.

\subsubsection{Considering impact}

By knowing the states of the CoM before impact $\boldsymbol{x}^{-*}$, the joint velocities 380$$
\dot{q}
$$
$\dot{\boldsymbol{q}}^{-*}$ are computed by means of 48 , with $\boldsymbol{\vartheta}^{-*}=\left[x^{-*}, y^{-*}, \boldsymbol{\phi}^{-\top^{*}}\right]^{\top}$ and $\dot{\vartheta}^{-*}=\left[\dot{x}^{-*}, \dot{y}^{-*}, \dot{\phi}^{-\top *}\right]^{\top}$ where $\phi^{-\top *}$ and $\dot{\phi}^{-\top *}$ are the external information at the end of the step. For instance for the case that $\phi=t, \phi^{-\top *}=T$ and $\dot{\phi}^{-T_{*}}=1$. Later, by using the transition model 4 the joint velocities after impact $\dot{\boldsymbol{q}}^{+}$are obtained. Then, the initial velocity of the generalized variables 385 after the transition $\dot{\boldsymbol{\xi}}\left(\boldsymbol{q}^{+}, \dot{\boldsymbol{q}}^{+}\right)$are deduced by means of 40 , namely, the initial velocity of the controlled variables and the CoM are obtained, i.e. $\dot{\boldsymbol{q}}_{c}\left(\boldsymbol{q}^{+}, \dot{\boldsymbol{q}}^{+}\right)$ and $\left(\dot{x}^{+*}, \dot{y}^{+*}\right)$. A diagram that explains the procedure for obtaining periodic walking gaits is shown in Fig. 3. 


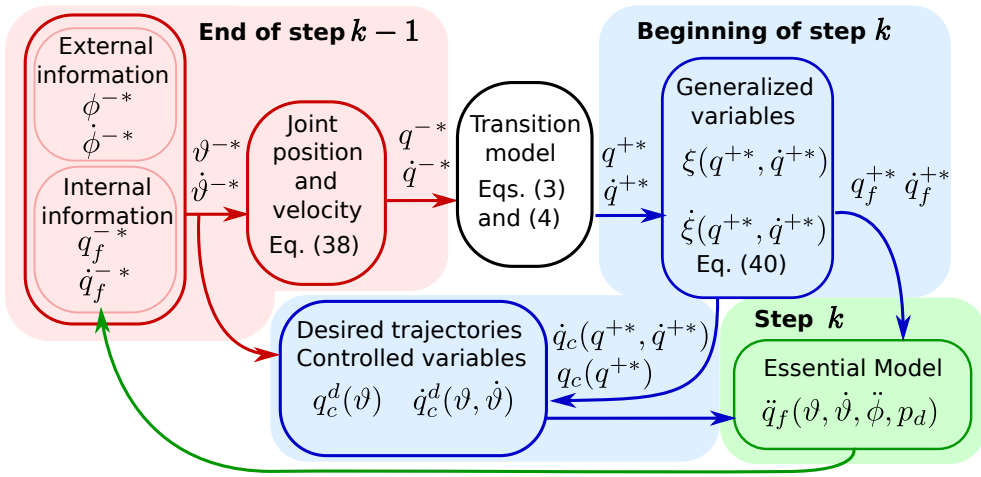

Figure 3: Periodic motion by using the essential model

\subsection{The desired motion of the swing foot and upper body}

The motion of the swing foot and upper body is defined by the desired controlled coordinates $\boldsymbol{q}_{c}^{d}(\boldsymbol{\vartheta})$. Since the essential model is developed under the assumption that the reference trajectories (38) are fulfilled for all the time, i.e.

$$
y \equiv \mathbf{0}, \quad \dot{y} \equiv \mathbf{0}, \quad \ddot{y} \equiv \mathbf{0}
$$
to interact with the environment can be performed by the arms while the robot walks. Therefore, depending on the complexity of the task the desired upperbody motion trajectories can be defined by simple polynomials or more complex 

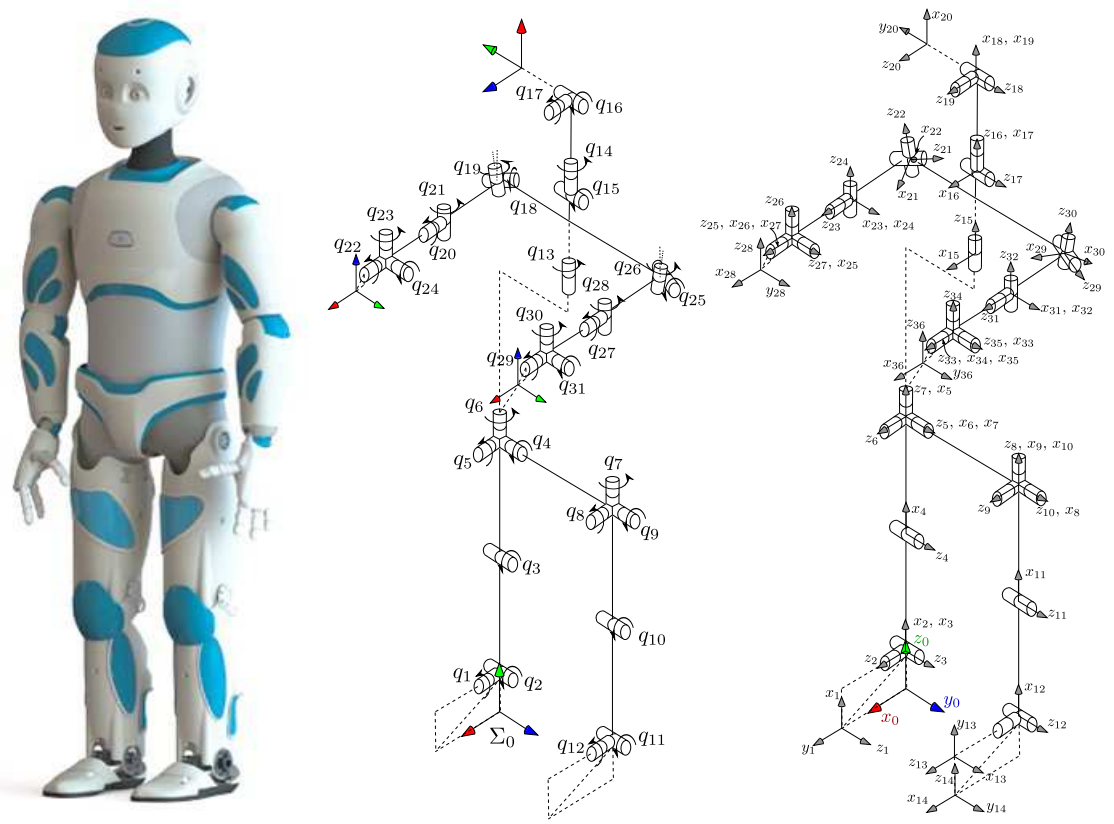

Figure 4: The humanoid robot ROMEO

functions.

\section{Case study: The humanoid robot ROMEO}

In this section, the essential model is exploited by taking into account the characteristics on the humanoid robot ROMEO. This robot has a weight of 40.8 $\mathrm{kg}$, a height of $1.46 \mathrm{~m}$ and it has $31 \mathrm{DoF}$ (6 DoF for each leg, $1 \mathrm{DOF}$ for the torso, 7 DOF for each arm and 4 DOF for the neck and head) as shown in Fig. 4. It has been developed by Softbank robotics [24.

In this paper, as explained in Section 4 the swing foot, the torso and the upper-body joints are chosen as points of interest. Thus, the configuration vector $\boldsymbol{\xi} \in \mathbb{R}^{31}$ is defined as in equation (37) where $\boldsymbol{q}_{c}(\boldsymbol{q})$ is chosen as $\boldsymbol{q}_{c}(\boldsymbol{q})=$ $\left[q_{c, 1}(\boldsymbol{q}), q_{c, 2}(\boldsymbol{q}), \cdots, q_{c, 29}(\boldsymbol{q})\right]^{\top}=\left[z(\boldsymbol{q}), x_{f}(\boldsymbol{q}), y_{f}(\boldsymbol{q}), z_{f}(\boldsymbol{q}), \psi_{f}(\boldsymbol{q}), \theta_{f}(\boldsymbol{q})\right.$ $\left.\varphi_{f}(\boldsymbol{q}), \psi_{t}(\boldsymbol{q}), \theta_{t}(\boldsymbol{q}), \varphi_{t}(\boldsymbol{q}), q_{13}, \ldots, q_{31}\right]^{\top}$, where $z$ is the height of the CoM of the robot, $x_{f}, y_{f}, z_{f}, \psi_{f}, \theta_{f}, \varphi_{f}$ are the position and orientation of the swing 
foot (roll, pitch and yaw) ${ }^{5}, \psi_{t}, \varphi_{t}$ are the orientation of the lower torso (the orientation of the hip, i.e. frame $\Sigma_{7}$ ). The upper-body joints $q_{13}$ to $q_{31}$ are the rest of controlled variables.

The desired trajectories for the 29 controlled variables as a function of vector $\boldsymbol{\vartheta}$ are defined as $\boldsymbol{q}_{c}^{d}(\boldsymbol{\vartheta})=\left[z(\boldsymbol{\vartheta}), x_{f, d}(\boldsymbol{\vartheta}), y_{f, d}(\boldsymbol{\vartheta}), z_{f, d}(\boldsymbol{\vartheta}), \psi_{f, d}(\boldsymbol{\vartheta}), \theta_{f, d}(\boldsymbol{\vartheta})\right.$, $\left.\varphi_{f, d}(\boldsymbol{\vartheta}), \psi_{t, d}(\boldsymbol{\vartheta}), \theta_{t, d}(\boldsymbol{\vartheta}), \varphi_{t, d}(\boldsymbol{\vartheta}), q_{d, 13}(\boldsymbol{\vartheta}), \ldots, q_{d, 31}(\boldsymbol{\vartheta})\right]^{\top}$.

Three different cases have been proposed to design the essential model in order to show its effectiveness.

1. Time trajectories are proposed to define the desired motion of the controlled variables $\boldsymbol{q}_{c}^{d}$. No impact is considered and a fixed upper-body pose and fixed location of the ZMP is desired for all the step. i.e. $\left(p_{x, d}, p_{y, d}\right)=$ $(0,0)$ w.r.t. $\Sigma_{0}$. Note that in here the inertial frame $\Sigma_{0}$ is attached to the ground under the support ankle (see Fig. 4). By considering a constant CoM vertical position, a comparison with the 3D LIP model is carried out.

2. Virtual constraints define the trajectories of the controlled variables $\boldsymbol{q}_{c}^{d}$ and an impact of the swing foot with the ground is considered to build an essential model where the upper-body and ZMP are kept in a fixed desired position as in the previous case.

3. A curve periodic motion is performed by specifying two different step parameters. Time trajectories are proposed to define the desired motion of the controlled variables $\boldsymbol{q}_{c}^{d}$. An impact of the swing foot with the ground is considered. Furthermore, an upper body motion and a desired trajectory for the ZMP is specified to be achieved during the step.

\subsection{Case I. The essential model closest to the 3D LIP model}

In order to show the efficiency of the essential model with respect to the 3D LIP model to generate walking gaits, in this section two walking gaits obtained by using these two models are compared. Since the 3D LIP model is a simplified

\footnotetext{
${ }^{5}$ Roll: turn around $\mathrm{x}$-axis. Pitch: turn around y-axis. Yaw: turn around z-axis.
} 
model of a general humanoid robot, it only considers the global CoM position of the robot, not its dynamics nor its parameters. On the other hand, the essential model takes into account the dynamical characteristics of the robot. Consequently, in order to compare the 3D LIP model and the essential model under the same conditions, it has been chosen:

- To consider a constant height of the CoM. Therefore, the gait parameters chosen for both models are shown in Table1.

- To consider a fixed step length, fixed step width and fixed step time to perform the gait.

- The evolution of the swing foot as functions of time in order to ensure the step is performed at a fixed step time.

- To define the trajectories of the swing foot by cycloidal motions in order to avoid impacts since the 3D LIP model does not consider them.

- To test the obtained periodic motions of both models in the complete model of ROMEO by considering the same swing foot motion and fixed upper body.

\subsubsection{The desired motion of the swing foot and upper body}

The evolution of the swing foot is given by

$$
\begin{aligned}
& x_{f, d}(t)=x_{f 0}+2 S\left[\frac{t}{T}-\frac{1}{2 \pi} \sin \left(\frac{2 \pi t}{T}\right)\right] \\
& y_{f, d}(t)=y_{f 0} \\
& z_{f, d}(t)= \begin{cases}h_{z}\left[\frac{2 t}{T}-\frac{1}{2 \pi} \sin \left(\frac{4 \pi t}{T}\right)\right] & \text { if } t \leq \frac{T}{2} \\
h_{z}-h_{z}\left[\frac{2 t-T}{T}-\frac{1}{2 \pi} \sin \left(\frac{4 \pi t}{T}\right)\right] & \text { if } t>\frac{T}{2}\end{cases}
\end{aligned}
$$

where $h_{z}[\mathrm{~m}]$ is the maximum desired height of the swing foot. As can be 465 seen, the initial and final value for $z_{f, d}$ is zero. The initial values of $x_{f 0}$ and $y_{f 0}$ are always measured with respect to the support foot, i.e. $\left[x_{f 0}, y_{f 0}\right]=[-S, D]$. 


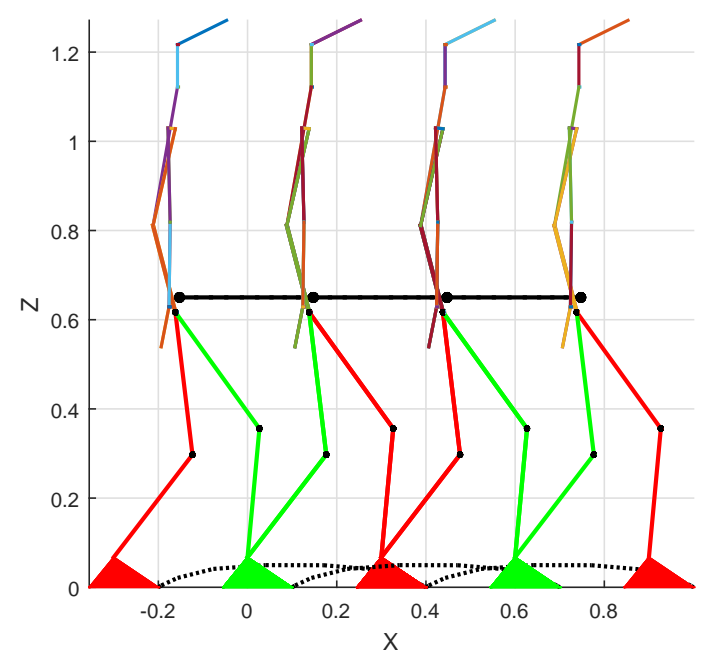

Figure 5: Sequence of steps performed by the robot ROMEO for the first case

Regarding the swing foot orientation and the upper body motion, it was decided to keep constant values. Therefore, the upper body is kept straight and the swing foot parallel to the ground during all the step.

\subsubsection{The essential model for ROMEO case 1}

In this case only time is used to define the desired trajectories for the controlled variables i.e. $\boldsymbol{q}_{c}^{d}(t)=\left[z_{0}, x_{f, d}(t), y_{f 0}, z_{f, d}(t), \boldsymbol{k}^{\top}\right]^{\top}$, where the elements of $\boldsymbol{k} \in \mathbb{R}^{25}$ are zero except for the ones in Table 2, Therefore, $\boldsymbol{\vartheta}=[x, y, t]^{\top}$ since $\boldsymbol{q}_{f}$ is always needed to define the joint motions. The desired position of the ZMP is $\left(p_{x, d}, p_{y, d}\right)=(0,0)$ w.r.t. $\Sigma_{0}$. Then, by following the procedure in Section 4.1 the evolution of the joints can be found as a function of $x, y$ and $t$. Later, by using the procedure in Section 3.3 the essential model is computed.

\subsubsection{Numerical comparison}

480

By using the gait parameters described previously, the periodic motion for 


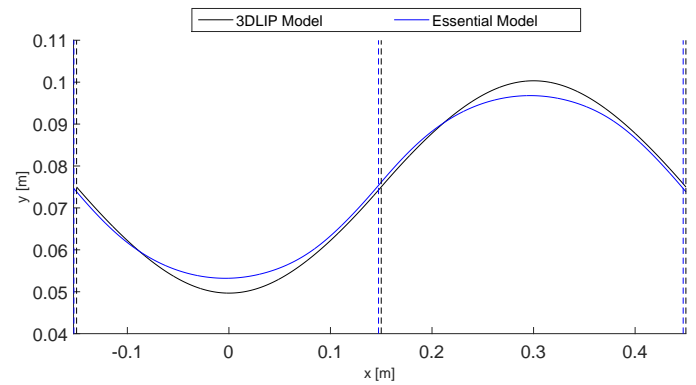

Figure 6: Comparison of the evolution of the CoM for the gaits obtained with the 3D LIP model and the essential model respectively.

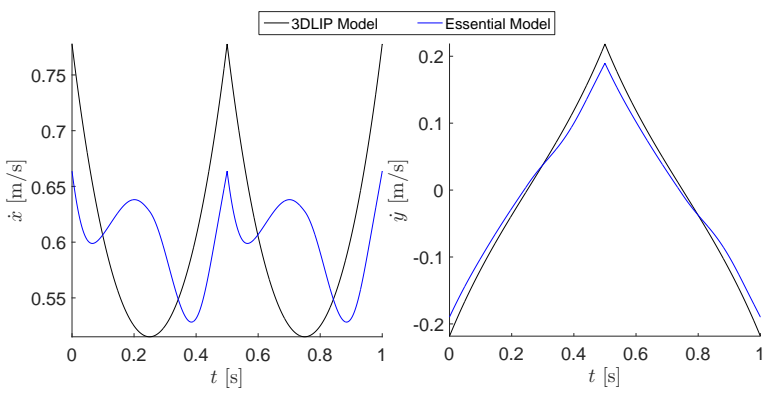

Figure 7: Comparison of the velocity of the CoM w.r.t. time for the gaits obtained with the 3D LIP model and the essential model respectively.

the 3D LIP model is defined by

$$
\boldsymbol{x}^{-*}=\left[\frac{S}{2}, \frac{D}{2}, \dot{x}^{-*}, \dot{y}^{-*}\right]^{\top}=[0.15,0.075,0.777764,0.218303]^{\top},
$$

meanwhile, the periodic motion for the essential model is defined by

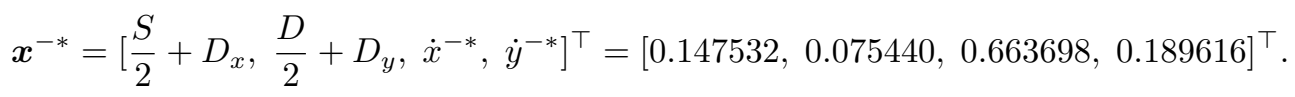

For this particular case, there is about $15 \%$ of difference between the velocities of each model in $X$ and $Y$ directions. The periodic motion for the essential model depends on the motion of the swing foot and upper body. However, the periodic motion of the 3D LIP model is a good initial guest to find periodic motions with the essential model.

For this case, the motion performed by the robot ROMEO for both models 


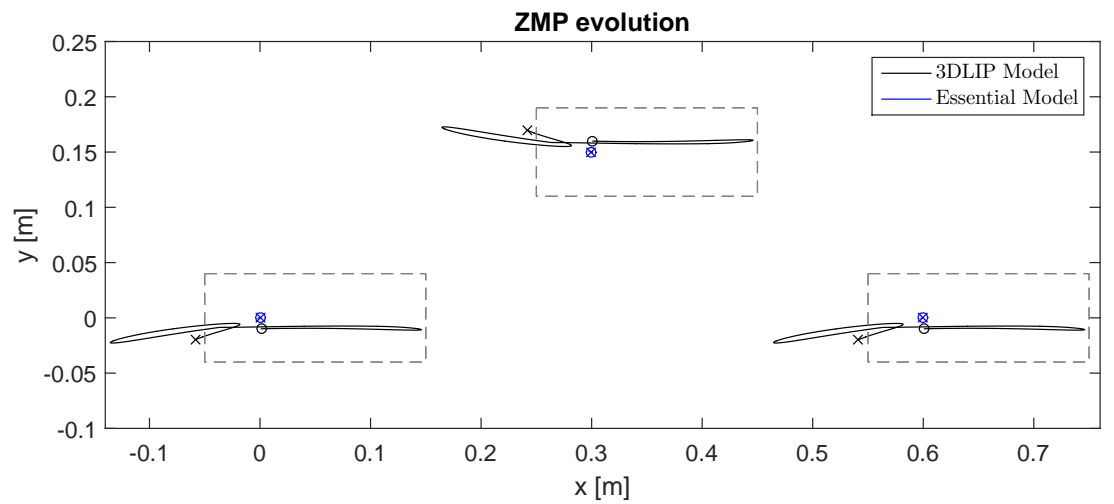

Figure 8: Comparison of the evolution of the ZMP for the gaits obtained with the 3D LIP model and the essential model respectively. It is shown how the gait obtained with the essential model makes the ZMP be kept in the desired location during all the walking gait. The circles and crosses denote the initial and final points of the ZMP respectively.

is very similar and it is illustrated in Fig. 5 where a simulation of three steps is shown. A video of the walking gait performed by ROMEO in simulation for Case I is attached to the paper. In order to see the difference between the two produced gaits Figs. 6 and 7 are plotted. Figure 6 shows that the spatial evolutions of the CoM of both models are close. In there, vertical dashed lines are used to indicate that a new step is performed (black for the 3D LIP model and blue for the essential model), and although they are very close, a small displacement $D_{x}$ is between them. On the other hand, the velocity of the CoM is plotted in Fig. 7. In here it can be noticed that the velocity of the CoM in $X$ direction must follow a complex function in order to fulfill the objective of keeping the ZMP in a desired location while the robot walks. Finally, Fig. 8 shows a comparison of the evolution of the ZMP by using the walking gaits obtained by each model. This plot clearly shows the advantage of the essential model. Most of the times, the 3D LIP model produces walking gaits where the ZMP evolves outside of the convex hull of support when they are replicated by the complete model of any humanoid robot. Therefore, some complementary techniques must be taken into account to adjust the evolution of the joints of the robot, in order to at least keep the ZMP into a place inside the convex 
Table 1: Gait parameters for the 3D LIP and Essential models

\begin{tabular}{|c|c|c|c|c|c|c|}
\hline \multirow{2}{*}{\multicolumn{2}{|c|}{$\begin{array}{c}\text { Parameter } \\
\text { [unit] }\end{array}$}} & \multicolumn{4}{|c|}{ Cases } & \multirow[t]{2}{*}{ Description } \\
\hline & & \multirow{2}{*}{$\begin{array}{c}\mathrm{I} \\
0.3\end{array}$} & \multirow{2}{*}{$\begin{array}{c}\text { II } \\
0.3\end{array}$} & \multirow{2}{*}{$\begin{array}{c}\mathrm{III}(\mathrm{S} 1) \\
0.3\end{array}$} & \multirow{2}{*}{$\frac{\mathrm{III}(\mathrm{S} 2)}{0.2653}$} & \\
\hline$S$ & {$[\mathrm{~m}]$} & & & & & Step length \\
\hline$D$ & {$[\mathrm{~m}]$} & 0.15 & 0.15 & 0.15 & 0.2436 & Step width \\
\hline$T$ & {$[\mathrm{~s}]$} & 0.5 & 0.5 & 0.5 & 0.5 & Step time \\
\hline$z_{0}$ & {$[\mathrm{~m}]$} & 0.65 & 0.65 & 0.65 & 0.65 & Height of the CoM \\
\hline$h_{z}$ & {$[\mathrm{~m}]$} & 0.05 & 0.05 & 0.05 & 0.05 & Max. swing foot amplitude \\
\hline$\varphi_{f, 0}$ & [deg] & 0 & 0 & 10 & -10 & Free foot initial rotation \\
\hline$\varphi_{f, f}$ & [deg] & 0 & 0 & -10 & 10 & Free foot final rotation \\
\hline$g$ & {$\left[\frac{m}{s^{2}}\right]$} & 9.81 & 9.81 & 9.81 & 9.81 & Gravity acceleration \\
\hline$\alpha_{z}$ & {$[\mathrm{~m}]$} & - & 0.05 & 0.05 & 0.05 & Max. CoM amplitude \\
\hline$v_{z}$ & $\left.\frac{m}{s}\right]$ & - & -0.2 & -0.2 & -0.2 & Desired landing velocity \\
\hline
\end{tabular}

505 However, in here, a simple evolution of the controlled variables is defined, i.e. only as a function of the position of the CoM in $X$ direction. In here, a step with similar characteristics to the previous case is desired. However, a different 
Table 2: Upper-body parameters for ROMEO

\begin{tabular}{cccccl}
\hline \hline$i$ & Case I & Case II & \multicolumn{2}{c}{ Case III } & Description \\
& $k_{i}$ & $k_{i}$ & $k_{0, i}$ & $k_{f, i}$ & {$[\mathrm{rad}]$} \\
\hline 7 & 0 & 0 & -0.25 & 0.25 & $q_{c, 11}^{d}$ Torso yaw \\
8 & 0 & 0 & 0.25 & -0.25 & $q_{c, 12}^{d}$ Neck yaw \\
12 & 1.7 & 0.41 & 1.8 & 1.6 & $q_{c, 16}^{d}$ R. shoulder pitch \\
13 & 0.2 & 0.19 & 0.2 & 0.2 & $q_{c, 17}^{d}$ R. shoulder yaw \\
14 & 0 & 0.194 & 1.9 & 1.9 & $q_{c, 18}^{d}$ R. elbow roll \\
15 & 0.05 & 1.523 & 0.3 & 0.3 & $q_{c, 19}^{d}$ R. elbow Yaw \\
18 & 0 & -0.415 & 0 & 0 & $q_{c, 22}^{d}$ R. wrist pitch \\
19 & 1.7 & 0.41 & 1.6 & 1.8 & $q_{c, 23}^{d}$ L. shoulder pitch \\
20 & -0.2 & -0.085 & -0.2 & -0.2 & $q_{c, 24}^{d}$ L. shoulder yaw \\
21 & 0 & -0.565 & -1.9 & -1.9 & $q_{c, 25}^{d}$ L. elbow roll \\
22 & -0.05 & -1.5 & -0.3 & -0.3 & $q_{c, 26}^{d}$ L. elbow yaw \\
24 & 0 & -0.165 & 0 & 0 & $q_{c, 27}^{d}$ L. wrist roll \\
25 & 0 & 0.32 & 0 & 0 & $q_{c, 29}^{d}$ L. wrist pitch \\
\hline
\end{tabular}
are shown in Tables 1 and 2 Moreover, an impact of the swing foot with the ground at transition is taken into account.

As explained in section 5.1.1 by knowing the states of the CoM before impact $\boldsymbol{x}^{-*}$ the states after impact $\boldsymbol{x}^{+*}$ can be deduced. Then, by taking into account 525 and $x^{-*}$, the trajectories of the controlled variables are computed.

\subsubsection{The evolution of the CoM}

In this case, a vertical displacement of the CoM is considered. This desired vertical motion of the CoM is defined by using a 5 th order polynomial function 
with the following boundary conditions

$$
\begin{array}{lll}
z_{d}\left(x^{+*}\right)=z_{0}, & z_{d}\left(x_{m}\right)=z_{0}+\alpha_{z}, & z_{d}\left(x^{-*}\right)=z_{0} \\
z_{d}^{\prime}\left(x^{+*}\right)=\frac{\dot{q}_{c, 1}}{\dot{x}^{+*}} & z_{d}^{\prime}\left(x_{m}\right)=0 & z_{d}^{\prime}\left(x^{-*}\right)=0
\end{array}
$$

where $\dot{q}_{c, 1}\left(\boldsymbol{q}^{+}, \dot{\boldsymbol{q}}^{+}\right)$is the vertical velocity of the CoM after impact 7 and $x^{+*}<$ ${ }_{530} x_{m}<x^{-*}$ is an intermediate desired point which in this case it is chosen exactly at the middle i.e. $x_{m}=D_{x}$.

\subsubsection{The desired motion of the swing foot and upper body}

It is desired that the swing foot land on the ground with a negative velocity. By doing this, the contact with the ground is ensured when the swing foot touches it. Therefore, as in the case of the vertical displacement of the CoM, a 5th order polynomial function is used to define the vertical evolution of the swing foot, by accomplishing the following boundary conditions

$$
\begin{aligned}
& z_{f, d}\left(x^{+*}\right)=0 \quad z_{f, d}\left(x_{m}\right)=h_{z} \quad z_{f, d}\left(x^{-*}\right)=0 \\
& z_{f, d}^{\prime}\left(x^{+*}\right)=\frac{\dot{q}_{c, 4}}{\dot{x}^{+*}} \quad z_{f, d}^{\prime}\left(x_{m}\right)=0 \quad z_{f, d}^{\prime}\left(x^{-*}\right)=\frac{v_{z}}{\dot{x}^{-*}}
\end{aligned}
$$

where $\dot{q}_{c, 4}\left(\boldsymbol{q}^{+}, \dot{\boldsymbol{q}}^{+}\right)$is the vertical velocity of the swing foot after impact ${ }^{8}$

For the horizontal motion of the swing foot, 3rd order polynomials are used,

\footnotetext{
${ }^{6}$ In here $z^{\prime}(x(t))=\frac{\partial}{\partial x} z(x(t))$, the same notation is used in the rest of the paper for the other variables.

${ }^{7} \dot{q}_{c, i}$ is the $i$-element of the derivative w.r.t. time of $\boldsymbol{q}_{c}$ which is defined at the beginning of Section 6

${ }^{8}$ Since there is a change of support, this velocity corresponds to the foot that was used as support in the previous step
} 
such as the following boundary conditions are fulfilled

$$
\begin{aligned}
x_{f, d}\left(x^{+*}\right) & =-S & x_{f, d}\left(x^{-*}\right) & =S \\
x_{f, d}^{\prime}\left(x^{+*}\right) & =\frac{\dot{q}_{c, 2}}{\dot{x}^{+*}} & x_{f, d}^{\prime}\left(x^{-*}\right) & =0 \\
y_{f, d}\left(x^{+*}\right) & =D & y_{f, d}\left(x^{-*}\right) & =D \\
y_{f, d}^{\prime}\left(x^{+*}\right) & =\frac{\dot{q}_{c, 3}}{\dot{x}^{+*}} & y_{f, d}^{\prime}\left(x^{-*}\right) & =0
\end{aligned}
$$

6.2.3. The essential model for ROMEO case 2 Table 2 .

$$
\begin{aligned}
q_{c, i}^{d}\left(x^{+*}\right) & =k_{i} & q_{c, i}^{d}\left(x^{-*}\right) & =k_{i} \\
q_{c, i}{ }^{\prime}{ }^{\prime}\left(x^{+*}\right) & =\frac{\dot{q}_{c, i}}{\dot{x}^{+*}} & q_{c, i}^{d}{ }^{\prime}\left(x^{-*}\right) & =0
\end{aligned}
$$

where $\dot{q}_{c, i}\left(\boldsymbol{q}^{+}, \dot{\boldsymbol{q}}^{+}\right)$is the velocity of the controlled variable $i$ after impact, and $k_{i}$ is the $i$-element of $\boldsymbol{k}$ whose elements are zero except for the ones defined in

In this case the trajectories for the controlled variables are defined as a function of $x$, i.e. $\boldsymbol{q}_{c}^{d}(x)=\left[z(x), x_{f, d}(x), y_{f, d}(x), z_{f, d}(x), q_{c, 5}(x), \cdots, q_{c, 29}(x)\right]^{\top}$, and $\boldsymbol{\vartheta}=\boldsymbol{q}_{f}=[x, y]^{\top}$. The desired position of the ZMP is $\left(p_{x, d}, p_{y, d}\right)=(0,0)$ 545

evious case. Then, by following the procedure in Section 4.1 the evolution of the joints can be found as a function of $x$ and $y$. Later, by using the procedure in Section 3.2 the essential model is computed.

\subsubsection{Numerical results}


By using the gait parameters shown in Table 1 and the upper-body configuration for ROMEO shown in Table 2 the periodic motion is defined by $\boldsymbol{x}^{-*}=\left[\frac{S}{2}+D_{x}, \frac{D}{2}+D_{y}, \dot{x}^{-*}, \dot{y}^{-*}\right]^{\top}=\left[\begin{array}{llll}0.153143, & 0.075467,0.67968,0.191304\end{array}\right]^{\top}$.

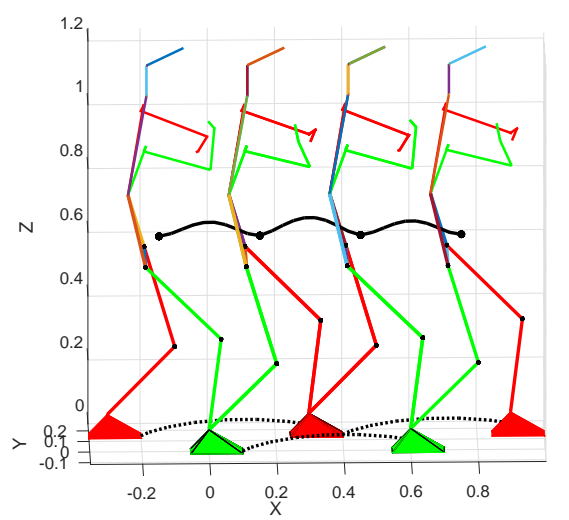

(a) Sequence of three steps

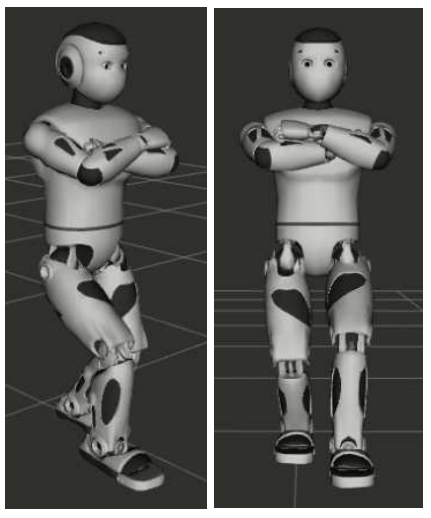

(b) 3D Model

Figure 9: Simulation performed by the robot ROMEO for the second case

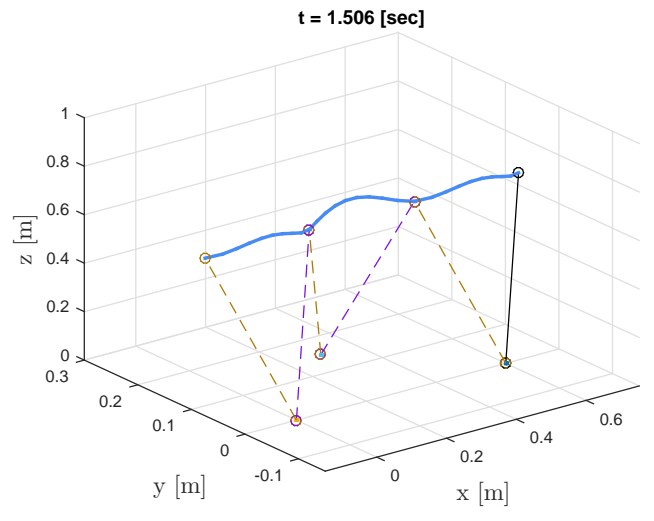

(a) Essential Model similar to the 3D IP

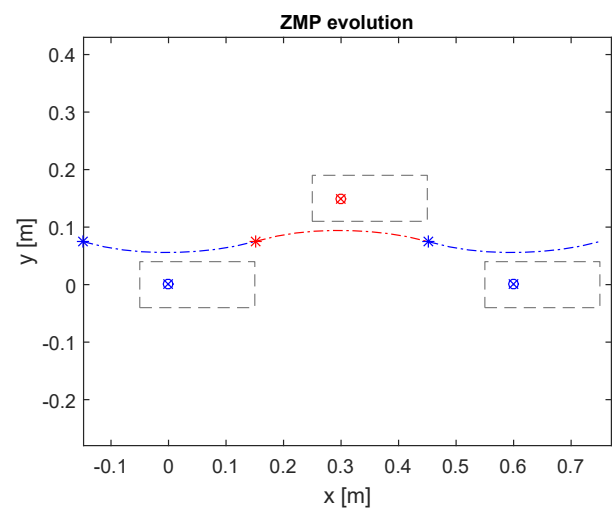

(b) ZMP evolution for each step

Figure 10: Essential model and footprints for the steps performed by the robot ROMEO for the second case

This walking gait holds the restriction of contact and no sliding, i.e. $F_{z}>0$ 
and $F_{\text {tan }}<F_{\text {fric }}$ by considering a friction coefficient $\mu=0.7$. A simulation of three steps performed by the robot ROMEO is shown in Fig. 9a. In there, the trajectory of the CoM is highlighted, where its oscillatory vertical motion is noted. By using a 3D model of the robot, the posture of the upper body is shown in Figure 9b. A video of the walking gait performed by ROMEO in simulation for this case is attached to the paper. Figure 10a shows the three steps performed by using the essential model, where the relation between the CoM and the ZMP is highlighted. Since a fixed ZMP position was imposed, the resulting simulation with the essential model looks very similar to an inverted pendulum model. However, as shown in Fig. 12b by generating the CoM trajectory with the essential model the ZMP is kept in a desired position at each step when the complete model is used (Fig. 9).

6.3. Case III. The essential model with impact, varying ZMP and upper body motion

In this last case, one of the main benefits of this model is exploited. We are referring to the development of a walking gait that tracks exactly a user-defined trajectory of the ZMP. Furthermore, by defining two different steps it is possible to achieve a periodic walking gait in a $3 \mathrm{D}$ space. In this example, a periodic walking gait while the robot turns is shown. The evolution of the controlled variables are also defined as a function of time, i.e. $\boldsymbol{q}_{c}^{d}(t)$, from $t=0$ to $t=T$. Nevertheless, unlike the first case of study, the impact of the swing foot with the ground is taken into account and a simple upper body motion is introduced.

The gait parameters for the first and second steps (S1 and S2 respectively) are shown in Table 1. And as mentioned earlier, in order to achieve a perfect tracking of the reference trajectories for all the time, feasible desired trajectories of the controlled coordinates must be designed. Therefore, as in the case two, the initial velocity after impact of the controlled variables, i.e. $\dot{\boldsymbol{h}}\left(\boldsymbol{q}^{+}, \dot{\boldsymbol{q}}^{+}\right)$, is again considered into the calculations of the desired trajectories $\boldsymbol{q}_{c}^{d}(t)$. 


\subsubsection{The desired evolution of the controlled variables}

The desired trajectories for the vertical motion of the CoM, the swing foot and the upper body are designed with polynomials as in the second case. Therefore, similar boundary conditions as (54), (55), (56), and (57) must be fulfilled. However, constant values $k_{i}$ for the desired initial and final positions of the upper body in equation (57) are replaced by $k_{0, i}$ and $k_{f, i}$ whose are defined in Table 2 Therefore, a simple motion of the upper body is performed, in particular a yaw-motion of the torso, a yaw-motion of the neck and a pitchmotion of both shoulders. Moreover, since in this case the trajectories are as functions of time, the polynomials are builded from 0 to $T$, instead of from $x^{+*}$ to $x^{-*}$ and the intermediate value has been chosen as $T / 2$ instead of $x_{m}$. Furthermore, the desired velocities $\dot{q}_{c, i}^{d}$ are computed directly instead of partial derivative $q_{c, i}^{d}$. Thereby, the boundary conditions 54, 55, 56, and (57) are used with desired initial velocities $\dot{q}_{c, i}^{d}(0)=\dot{q}_{c, i}\left(\boldsymbol{q}^{+}, \dot{\boldsymbol{q}}^{+}\right)$for $i=1, \ldots, 29$ and desired final velocities zero except for the one of the swing foot, which is ${ }_{595} \quad \dot{q}_{c, 4}^{d}(T)=\dot{z}_{f, d}(T)=v_{z}$.

\subsubsection{The desired motion of the $Z M P$}

Unlike the two previous cases where a desired fix position of the ZMP was chosen, in here it is proposed to define a trajectory for the ZMP while the robot perform a step. Several studies about the evolution of ZMP on a human walking gait, such as [25, 26, 27] can be used with the essential model in order to develop human-like walking gaits.

In this case, 3rd and 4th order polynomials functions are used in order to build a soft desired trajectory of the ZMP in $X$ and $Y$ direction respectively, such as the following boundary conditions are fulfilled

$$
\begin{array}{ll}
p_{x, d}(0)=p_{x 0} & p_{x, d}(T)=p_{x f} \\
\dot{p}_{x, d}(0)=0 & \dot{p}_{x, d}(T)=0,
\end{array}
$$


and

$$
\begin{aligned}
& p_{y, d}(0)=p_{y 0} \quad p_{y, d}(T / 2)=p_{y m} \quad p_{y, d}(T)=p_{y f} \\
& \dot{p}_{y, d}(0)=0 \quad \dot{p}_{y, d}(T)=0
\end{aligned}
$$

where $p_{x 0}=-0.02[\mathrm{~m}], p_{x f}=-0.10[\mathrm{~m}]$, are the initial and final desired positions of the ZMP in $X$ direction w.r.t. the support frame $\Sigma_{0}$, and $p_{y 0}=$ $p_{y f}=0.01[\mathrm{~m}], p_{y m}=0[\mathrm{~m}]$, are the initial, final and intermediate positions of the ZMP in $Y$ direction w.r.t. the frame $\Sigma_{0}$.

\subsubsection{The essential model for ROMEO case 3}

In this case the trajectories for the controlled variables are defined as a function of time, i.e. $\boldsymbol{q}_{c}^{d}(t)=\left[z(t), x_{f, d}(t), y_{f, d}(t), z_{f, d}(t), q_{c, 5}(t), \cdots, q_{c, 29}(t)\right]^{\top}$, and $\boldsymbol{\vartheta}=\boldsymbol{q}_{f}=[x, y, t]^{\top}$. The polynomials $p_{x, d}(t)$ and $p_{y, d}(t)$ define the desired trajectory of the ZMP in $X$ and $Y$ direction w.r.t. $\Sigma_{0}$. Then, by following the procedure in Section 4.1 the evolution of the joints can be found as a function of $x, y$ and $t$. Later, by using the procedure in Section 3.3 the essential model 615 is computed.

\subsubsection{Numerical results}

By using the gait parameters shown in Table 1 and the upper-body configuration for ROMEO shown in Table 2 the periodic motion is defined by $\boldsymbol{x}^{-*}=\left[\frac{S}{2}+D_{x}, \frac{D}{2}+D_{y}, \dot{x}^{-*}, \dot{y}^{-*}\right]^{\top}=[0.1936,0.074921,0.583253,0.174165]^{\top}$

As in the second case, the restriction of contact and no sliding is ensured, i.e. $F_{z}>0$ and $F_{\text {tan }}<F_{\text {fric }}$ by considering a friction coefficient $\mu=0.7$. 620 A simulation of twelve steps performed by the robot ROMEO is shown in Fig. 11a. In that figure, the trajectory of the CoM is highlighted, where its oscillatory vertical motion is noted. A sequence of a $3 \mathrm{D}$ model of the robot performing the periodic gait while turning is shown in Figure 11b. A video of the walking 


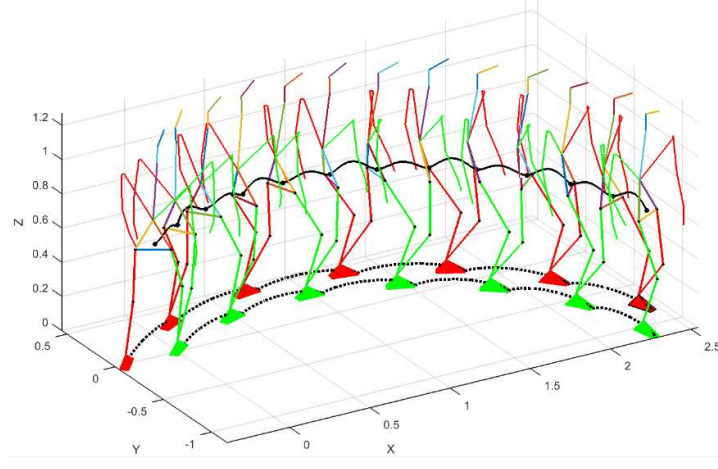

(a) Sequence of twelve steps

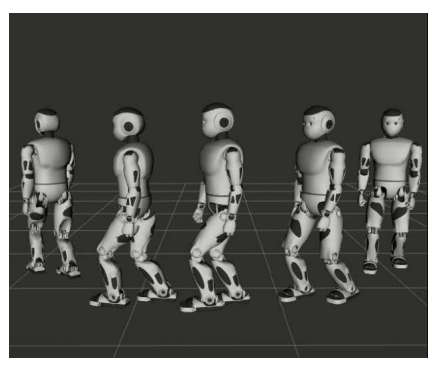

(b) 3D Model

Figure 11: Simulation performed by the robot ROMEO for the third case

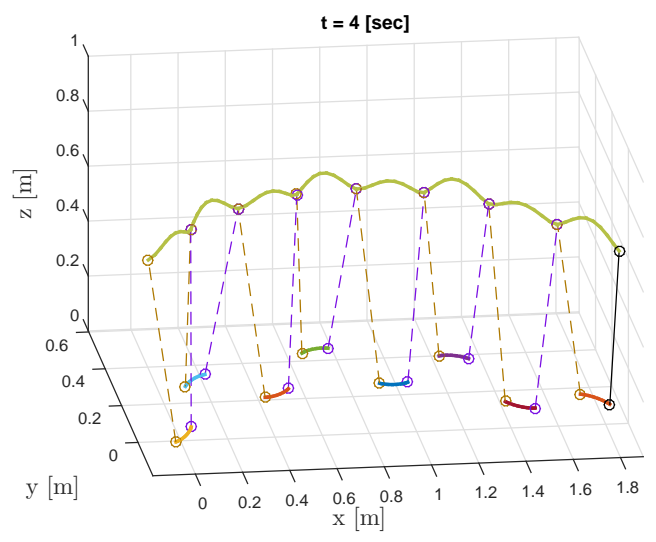

(a) Essential Model similar to the 3D IP

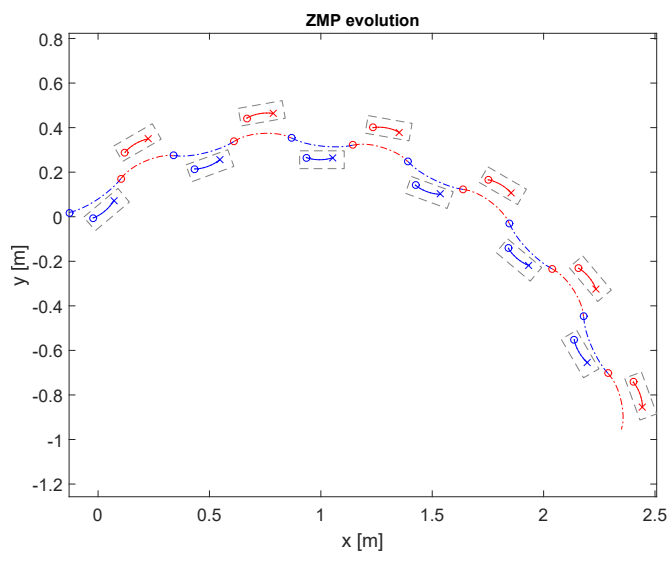

(b) ZMP evolution for each step

Figure 12: Essential model and footprints for the steps performed by the robot ROMEO for the third case

gait performed by ROMEO in simulation for Case III is attached to the paper.

Figure 12a shows the first eight steps using the essential model. In there, the relation between the $\mathrm{CoM}$ and the ZMP is highlighted. Notice that, since the variable ZMP trajectory can be imposed, this relation could be seen as an inverted pendulum with a mobil base. In the Fig. $12 \mathrm{~b}$ it is shown the footprints of the support foot for the twelve steps. In each footprint, it can be noted that the evolution of the ZMP follows the desired trajectory. 


\section{Conclusion}

The use of simple models, as the 3D LIP model, for building walking trajectories are useful. However, the vertical motion of the CoM is constrained and the ZMP is not keep to its desired location when the complete dynamics inside the convex hull of support are usually carried out. In this paper, a new model of the same dimension as the 3D LIP model, called essential model, has been proposed in order to deal with these difficulties. This model is developed by taking into account the whole dynamics of the robot and its simplification 640 a consequence, the resulting walking gaits always keep the ZMP in the desired path. Furthermore, impacts of the swing foot with the ground can also be considered in the development of walking gaits. The dynamics of the essential model depends always on the internal states of the robot but can also depends be used to develop motions in double support phases, however in this paper, only a continuous single support and an instantaneous double support phases are taken into account. By using the robot ROMEO as a case of study, three essential models where designed in order to show the efficiency of this proposal. gaits with better performance. Some future works on this issue are:

- Using the essential model to propose starting and stopping phases.

- Exploiting this model for the motion of the robot during double support or multi-contact phases.

655
- Introducing optimization to the upper body motion in order to reduce the joint torques.

- Proposing human-like trajectories for the ZMP in order to develop more efficient walking gaits. 
- Introducing different external information to define the desired trajectories of the controlled variables, not just the time. This external information could be the CoM of other robots in order to achieve walking synchronization among robots, or joysticks to handle the motion of the robot as a function of them, etc.

\section{Acknowledgements}

The authors are grateful with the CNRS (France) and CONACyT (México) for the support received by means of the ANR Robotex project and a postdoctoral grant respectively.

\section{Appendix. Numerical computing of $A_{\vartheta}$ and $d_{\vartheta}$.}

According with equation $(7)$ or $(9)$ by using $\boldsymbol{q}, \dot{\boldsymbol{q}}$ and $\ddot{\boldsymbol{q}}$ as inputs of the

Newton-Euler algorithm, the reaction force, reaction moment and joint torques can be computed. This method can be also utilized to compute $\ddot{\boldsymbol{q}}_{f}$. In order to achieve this, let us take advantage of the linearity in the acceleration in (9) and give as input of the NE algorithm the acceleration $\ddot{\boldsymbol{q}}$ "by parts". With this proposal, the NE algorithm will be performed $m+3$ times as shown below.

Notice that equation 25) could be written as

$$
\ddot{\boldsymbol{q}}=J_{\vartheta(:, 1)} \ddot{x}+J_{\vartheta(:, 2)} \ddot{y}+J_{\vartheta(:, 3)} \ddot{\phi}_{1}+\cdots+J_{\vartheta(:, m+2)} \ddot{\phi}_{m}+\dot{J}_{\vartheta} \dot{\boldsymbol{\vartheta}}
$$

where $J_{\vartheta(:, i)}$ means the $i$-th column of matrix $J_{\vartheta}$.

Thus, considering $\ddot{\boldsymbol{q}}$ from equation A.1 and by giving $\boldsymbol{\Delta}_{i}=J_{\vartheta(:, i)}+\dot{J}_{\vartheta} \dot{\boldsymbol{\vartheta}}$ into equation $(9)$ instead of $\ddot{\boldsymbol{q}}$ we will get

$$
\boldsymbol{N} \boldsymbol{E}_{i}=A_{e}(\boldsymbol{\vartheta})\left[J_{\vartheta(:, i)}+\dot{J}_{\vartheta} \dot{\boldsymbol{\vartheta}}\right]+\boldsymbol{d}_{e}(\boldsymbol{\vartheta}, \dot{\boldsymbol{\vartheta}}) .
$$

for $i=1,2, \ldots, m+2$. Then, by giving $\boldsymbol{\Delta}_{m+3}=\dot{J}_{\vartheta} \dot{\boldsymbol{\vartheta}}$ instead $\ddot{\boldsymbol{q}}$ into equation $680 \quad(9)$ we get

$$
\boldsymbol{N} \boldsymbol{E}_{m+3}=A_{e} \dot{J}_{\vartheta} \dot{\boldsymbol{\vartheta}}+\boldsymbol{d}_{e}
$$


Then, by using equations $\mathrm{A} .2$ and $\mathrm{A} .3$ we can recover 26 and finally 27 as follows

$$
\begin{aligned}
& \boldsymbol{N E}=\left[\boldsymbol{N} \boldsymbol{E}_{1}-\boldsymbol{N} \boldsymbol{E}_{m+3}\right] \ddot{x}+\left[\boldsymbol{N} \boldsymbol{E}_{2}-\boldsymbol{N} \boldsymbol{E}_{m+3}\right] \ddot{y}+ \\
& {\left[\boldsymbol{N} \boldsymbol{E}_{3}-\boldsymbol{N} \boldsymbol{E}_{m+3}\right] \ddot{\phi}_{1}+\ldots+\left[\boldsymbol{N} \boldsymbol{E}_{m+2}-\boldsymbol{N} \boldsymbol{E}_{m+3}\right] \ddot{\phi}_{m}+\boldsymbol{N} \boldsymbol{E}_{m+3}} \\
& =\left[A_{e}\left[J_{\vartheta(:, 1)}+\dot{J}_{\vartheta} \dot{\boldsymbol{\vartheta}}\right]+\boldsymbol{d}_{e}-A_{e} \dot{J}_{\vartheta} \dot{\boldsymbol{\vartheta}}-\boldsymbol{d}_{e}\right] \ddot{x}+ \\
& {\left[A_{e}\left[J_{\vartheta(:, 2)}+\dot{J}_{\vartheta} \dot{\boldsymbol{\vartheta}}\right]+\boldsymbol{d}_{e}-A_{e} \dot{J}_{\vartheta} \dot{\boldsymbol{\vartheta}}-\boldsymbol{d}_{e}\right] \ddot{y}+} \\
& {\left[A_{e}\left[J_{\vartheta(:, 3)}+\dot{J}_{\vartheta} \dot{\boldsymbol{\vartheta}}\right]+\boldsymbol{d}_{e}-A_{e} \dot{J}_{\vartheta} \dot{\boldsymbol{\vartheta}}-\boldsymbol{d}_{e}\right] \ddot{\phi}_{1}+\ldots} \\
& +\left[A_{e}\left[J_{\vartheta(:, m+2)}+\dot{J}_{\vartheta} \dot{\boldsymbol{\vartheta}}\right]+\boldsymbol{d}_{e}-A_{e} \dot{J}_{\vartheta} \dot{\boldsymbol{\vartheta}}-\boldsymbol{d}_{e}\right] \ddot{\phi}_{m}+A_{e} \dot{J}_{\vartheta} \dot{\boldsymbol{\vartheta}}+\boldsymbol{d}_{e} \\
& =A_{e} J_{\vartheta(:, 1)} \ddot{x}+A_{e} J_{\vartheta(:, 2)} \ddot{y}+A_{e} J_{\vartheta(:, 3)} \ddot{\phi}_{1}+\ldots+A_{e} J_{\vartheta(:, m+2)} \ddot{\phi}_{m}+A_{e} \dot{J}_{\vartheta} \dot{\boldsymbol{\vartheta}}+\boldsymbol{d}_{e} \\
& =A_{e}\left[J_{\vartheta(:, 1)}, J_{\vartheta(:, 2)}, J_{\vartheta(:, 3)}, \cdots, J_{\vartheta(:, m+2)}\right]\left[\begin{array}{c}
\ddot{x} \\
\ddot{y} \\
\ddot{\phi}_{1} \\
\vdots \\
\ddot{\phi}_{m}
\end{array}\right]+A_{e} \dot{J}_{\vartheta} \dot{\boldsymbol{\vartheta}}+\boldsymbol{d}_{e} \\
& =A_{e} J_{\vartheta} \ddot{\vartheta}+A_{e} \dot{J}_{\vartheta} \dot{\boldsymbol{\vartheta}}+\boldsymbol{d}_{e} \\
& =A_{\vartheta} \ddot{\boldsymbol{\vartheta}}+\boldsymbol{d}_{\vartheta}
\end{aligned}
$$

where it is shown that

$$
\begin{aligned}
& A_{\vartheta}=\left[\boldsymbol{N} \boldsymbol{E}_{1}-\boldsymbol{N} \boldsymbol{E}_{m+3}, \quad \boldsymbol{N} \boldsymbol{E}_{2}-\boldsymbol{N} \boldsymbol{E}_{m+3},\right. \\
& \left.\boldsymbol{N} \boldsymbol{E}_{3}-\boldsymbol{N} \boldsymbol{E}_{m+3}, \quad \cdots, \quad \boldsymbol{N} \boldsymbol{E}_{m+2}-\boldsymbol{N} \boldsymbol{E}_{m+3}\right] \\
& \boldsymbol{d}_{\vartheta}=\boldsymbol{N} \boldsymbol{E}_{m+3}
\end{aligned}
$$

Notice that the construction of matrix $A_{\vartheta}$ is not required, but the product $\left[\boldsymbol{N} \boldsymbol{E}_{j+2}-\boldsymbol{N} \boldsymbol{E}_{m+3}\right] \ddot{\phi}_{j}$, with $j=1, \ldots, m$. Thus, if the element $\ddot{\phi}_{j}$ of $\ddot{\phi}$ is zero, $\left[\boldsymbol{N} \boldsymbol{E}_{j+2}-\boldsymbol{N} \boldsymbol{E}_{m+3}\right] \ddot{\phi}_{j}=0$, thus, the use of the NE algorithm can be avoided for that particular iteration saving time of computation. 


\section{References}

[1] J. Englsberger, C. Ott, A. Albu-Sch affer, Three-dimensional bipedal walking control based on divergent component of motion, IEEE Transactions on Robotics 31 (2) (2015) 355-368.

[2] J. Englsberger, C. Ott, M. A. Roa, A. Albu-Sch affer, G. Hirzinger, Bipedal walking control based on capture point dynamics, in: 2011 IEEE/RSJ Int. Conf. on Intelligent Robots and Systems, 2011, pp. 4420-4427.

[3] S. Kajita, M. Benallegue, R. Cisneros, T. Sakaguchi, S. Nakaoka, M. Morisawa, K. Kaneko, F. Kanehiro, Biped walking pattern generation based on spatially quantized dynamics, in: IEEE-RAS 17th International Conference on Humanoid Robotics (Humanoids), 2017, pp. 599-605.

[4] L. Zhang, C. Zhou, Optimal three-dimensional biped walking pattern generation based on geodesics, International Journal of Advanced Robotic Systems 14 (2) (2017) 1-11.

[5] M. Vukobratović, B. Borovac, Zero-moment point - thirty five years of its life, International Journal of Humanoid Robotics 1 (1) (2004) 157-173.

[6] S. Kajita, F. Kanehiro, K. Kaneko, K. Fujiwara, K. Harada, K. Yokoi, H. Hirukawa, Biped walking pattern generation by using preview control of zero-moment point, in: 2003 IEEE Int. Conf. on Robotics and Automation (Cat. No.03CH37422), Vol. 2, 2003, pp. 1620-1626.

[7] C. Chevallereau, G. Abba, Y. Aoustin, E. R. W. F. Plestan, C. Canudas, J. Grizzle, Rabbit: a testbed for advanced control theory, IEEE Control Systems Magazine 23(5) (2003) 57-79.

[8] C. Chevallereau, Y. Aoustin, Self-stabilization of 3d walking via vertical oscillations of the hip, in: 2015 IEEE Int. Conf. on Robotics and Automation (ICRA), 2015, pp. 5088-5093. 
[9] B. Griffin, J. Grizzle, Nonholonomic virtual constraints and gait optimization for robust walking control, The International Journal of Robotics Research 36 (8) (2017) 895-922.

[10] A. D. Ames, P. Tabuada, A. Jones, W.-L. Ma, M. Rungger, B. Schürmann, S. Kolathaya, J. W. Grizzle, First steps toward formal controller synthesis for bipedal robots with experimental implementation, Nonlinear Analysis: Hybrid Systems 25 (2017) 155 - 173.

[11] C. Chevallereau, H. Razavi, D. Six, Y. Aoustin, J. Grizzle, Selfsynchronization and self-stabilization of 3d bipedal walking gaits, Robotics and Autonomous Systems 100 (Supplement C) (2018) 43 - 60.

[12] T. Saidouni, G. Bessonnet, Generating globally optimised sagittal gait cycles of a biped robot, Robotica 21 (2) (2003) 199-210.

[13] D. Tlalolini, Y. Aoustin, C. Chevallereau, Design of a walking cyclic gait with single support phases and impacts for the locomotor system of a thirteen-link 3d biped using the parametric optimization, Multibody Syst Dyn 23 (2010) 33-56.

[14] V. De-León-Gómez, J. A. Pámanes, V. Santibanez, Experimental evaluation of the real-time walking of a 5 dof biped robot with a gait based on cycloidal motions, Revista Iberoamericana de Automática e Informática Industrial RIAI 12 (04) (2015) 408-418.

[15] A. Isidori, The zero dynamics of a nonlinear system: From the origin to the latest progresses of a long successful story 19 (2011) 18 - 25.

[16] T. Wang, C. Chevallereau, Stability analysis and time-varying walking control for an under-actuated planar biped robot, Robotics and Autonomous Systems 59 (6) (2011) 444.

[17] J. W. Grizzle, C. Chevallereau, Virtual Constraints and Hybrid Zero Dynamics for Realizing Underactuated Bipedal Locomotion, Springer Netherlands, Dordrecht, 2018, pp. 1-31. 
[18] H. Razavi, A. M. Bloch, C. Chevallereau, J. W. Grizzle, Restricted discrete invariance and self-synchronization for stable walking of bipedal robots, in: 2015 American Control Conference (ACC), 2015, pp. 4818-4824.

[19] V. De-León-Gómez, V. Santibanez, J. Moreno-Valenzuela, A procedure to find equivalences among dynamic models of planar biped robots, Simulation Modelling Practice and Theory 75 (2017) $48-66$.

[20] D. E. Orin, A. Goswami, S.-H. Lee, Centroidal dynamics of a humanoid robot, Autonomous Robots 35 (2) (2013) 161-176.

[21] S. Kajita, F. Kanehiro, K. Kaneko, K. Yokoi, H. Hirukawa, The 3d linear inverted pendulum mode: A simple modeling for a biped walking pattern generation, in: the 2001 IEEE/RSJ Int. Conf. on Intelligent Robots and Systems, Maui, HI, USA, 2001, pp. 239-246.

[22] J. Romkes, K. Bracht-Schweizer, The effects of walking speed on upper body kinematics during gait in healthy subjects, Gait \& Posture 54 (2017) 304-310.

[23] J. Liu, U. Schwiegelshohn, O. Urbann, Stable walking of a bipedal humanoid robot involving three-dimensional upper body motion, in: 2014 IEEE-RAS Int. Conf. on Humanoid Robots, 2014, pp. 80-85.

[24] SoftBank Group. Aldebaran-Robotics. Project ROMEO, https://www. ald.softbankrobotics.com/en, http://projetromeo.com/, accessed: 2018-04-15 (2009-2016, Paris, France.).

[25] I. Danilov, B. Gabbasov, I. Afanasyev, E. Magid, Zmp trajectory from human body locomotion dynamics evaluated by kinect-based motion capture system, in: Int. Conf. on Computer Vision Theory and Applications (VISAPP), Rome, Italy, 2016, pp. 160-166.

[26] X. Zhang, H. Wang, Y. Shi, C. Fu, H. Wang, G. Wang, A measure system of zero moment point using wearable inertial sensors, China Communications 13 (5) (2016) 16-27. 
[27] Y. Aoustin, A. M. Formalskii, Walking of biped with passive exoskeleton: evaluation of energy consumption, Multibody System Dynamics 43 (1)

(2018) 71-96. doi:10.1007/s11044-017-9602-7. 